\title{
GENERALIZED MCKAY QUIVERS, ROOT SYSTEM AND KAC-MOODY ALGEBRAS
}

\author{
Bo Hou And Shilin Yang
}

\begin{abstract}
Let $Q$ be a finite quiver and $G \subseteq \operatorname{Aut}(\mathbb{k} Q)$ a finite abelian group. Assume that $\widehat{Q}$ and $\Gamma$ are the generalized Mckay quiver and the valued graph corresponding to $(Q, G)$ respectively. In this paper we discuss the relationship between indecomposable $\widehat{Q}$-representations and the root system of Kac-Moody algebra $\mathfrak{g}(\Gamma)$. Moreover, we may lift $G$ to $\bar{G} \subseteq \operatorname{Aut}(\mathfrak{g}(\widehat{Q}))$ such that $\mathfrak{g}(\Gamma)$ embeds into the fixed point algebra $\mathfrak{g}(\widehat{Q})^{\bar{G}}$ and $\mathfrak{g}(\widehat{Q})^{\bar{G}}$ as a $\mathfrak{g}(\Gamma)$-module is integrable.
\end{abstract}

\section{Introduction}

Thirty years ago, McKay introduced a class of quivers, now called the McKay quivers, for some finite subgroups of the general linear group [16]. Let $\mathbb{C}$ denote the complex number field. McKay observed that the McKay quivers for the subgroups of $\operatorname{SL}(2, \mathbb{C})$ are the double quivers of the extended Dynkin quivers $\widetilde{A}_{n}, \widetilde{D}_{n}, \widetilde{E}_{6}, \widetilde{E}_{7}, \widetilde{E}_{8}$. McKay quiver has played an important role in many mathematical fields such as quantum group, algebraic geometry, mathematics physics and representation theory (see, for examples $[2,4,8,9,15,17]$ ).

Let $V$ be a finite vector space over an algebraically closed field $\mathbb{k}$ of characteristic 0 and $G \subseteq \mathrm{GL}_{\mathbb{k}}(V)$ a finite group. Assume that $\mathrm{T}_{\mathrm{k}}(V)$ is the tensor algebra of $V$ over $\mathbb{k}$. It is well-known that the skew group algebra $\mathrm{T}_{\mathrm{k}}(V) * G$ is Morita equivalent to the path algebra $\mathbb{k} \widehat{Q}$, where $\widehat{Q}$ is the McKay quiver of $G$ (see [9]). In other words, the McKay quiver realizes the Gabriel quiver of $\mathrm{T}_{\mathrm{k}}(V) * G$. It is natural to ask how to determine the Gabriel quiver of skew group algebra $\Lambda * G$ for any algebra $\Lambda$. Recently, for arbitrary path algebra $\mathbb{k} Q$ over an algebraically closed field $\mathbb{k}$ and a finite group $G$ such that chark $\nmid|G|$, if the action of $G$ on $\mathbb{k} Q$ permutes the set of primitive idempotents and stabilizing the vector space spanned by the arrows, Demonet in [5] has constructed a

Received December 17, 2013; Revised December 12, 2014.

2010 Mathematics Subject Classification. Primary 16G10, 16G20, 17B67.

Key words and phrases. generalized McKay quiver, representation of quiver, root system, Kac-Moody algebra.

The authors are supported by the National Natural Science Foundation of China (Grant. 11301144, 11271043, 11471186). 
quiver $\widehat{Q}$ such that the path algebra $\mathbb{k} \widehat{Q}$ is Morita equivalent to the skew group algebra $\mathbb{k} Q * G$. The quiver $\widehat{Q}$ can be viewed as a generalization of McKay quiver, which is called the generalized McKay quiver of $(Q, G)$ in this paper.

Given a finite quiver $Q$ with an admissible automorphism a. Hubery in $[11,12]$ described the correspondence between dimension vectors of the isomorphically invariant $Q$-indecomposables and the positive root system of $\mathfrak{g}(\Gamma)$, where $\Gamma$ is the valued graph of $(Q, \mathbf{a})$. Deng, Du, et al. proved that the similar correspondence between representations of the species of $(Q, \mathbf{a})$ over finite field and the positive root system of $\mathfrak{g}(\Gamma)$ by Frobenius morphism [6]. Motivated by Hubery's work, the aim of this paper is to establish the correspondence between the indecomposable $\widehat{Q}$-representations and the positive roots of the symmetrizable Kac-Moody algebra $\mathfrak{g}(\Gamma)$ of the valued graph $\Gamma$ associated to $(Q, G)$, where $Q$ is a finite quiver and $G$ is a finite abelian automorphism group of $\mathbb{k} Q$. Moreover, we can lift $G$ to an automorphism group $\bar{G}$ of Kac-Moody algebra $\mathfrak{g}:=\mathfrak{g}(\widehat{Q})$ of $\widehat{Q}$, such that $\mathfrak{g}(\Gamma)$ can be embedded into the fixed point subalgebra $\mathfrak{g}^{\bar{G}}$. In this case, we also show that $\mathfrak{g}^{\bar{G}}$ as a $\mathfrak{g}(\Gamma)$-module is integrable. Compared with Hubery's work, a more general description is given by approach of the generalized McKay quiver.

For a finite quiver $Q=(I, E)$ and a finite abelian group $G \subseteq \operatorname{Aut}(\mathbb{k} Q)$ (the algebra automorphism group of $\mathbb{k} Q$ ). We always assume that the action of $G$ on $Q$ is admissible, i.e., no arrow connects vertices in the same $G$-orbit. Then we can get a valued graph $\Gamma$ without loops and a generalized McKay quiver $\widehat{Q}$ corresponding to $(Q, G)$. By [18], we can define an action of $G$ on $\mathbb{k} \widehat{Q}$ due to the Morita equivalence between the skew group algebra $\mathbb{k} Q * G$ and $\mathbb{k} \widehat{Q}$. Therefore this action induces an action on $\widehat{Q}$-representations. Let $G_{X}$ be a complete set of left coset representatives of $H_{X}=\left\{g \in G \mid{ }^{g} X \cong X\right\}$ in $G$ for any $\widehat{Q}$-representation $X$, let $\mathbb{Z} I, \mathbb{Z} \widehat{I}$ and $\mathbb{Z I}$ be the root lattice of $Q, \widehat{Q}$ and $\Gamma$, respectively. Applying the equivalence between representation category of $\widehat{Q}$ and module category of the skew group algebra $\mathbb{k} Q * G$ and the fact that each $\mathbb{k} Q * G$ module as a $Q$-representation is $G$-invariant, we define a map

$$
h: \mathbb{Z} \widehat{I} \longrightarrow(\mathbb{Z} I)^{G} \longrightarrow \mathbb{Z I}
$$

where $(\mathbb{Z} I)^{G}$ is the fixed point set of $\mathbb{Z} I$ under the action of $G$. The map $h$ builds a bridge between the dimension vectors of indecomposable $\widehat{Q}$-representations and the root system of Kac-Moody algebra $\mathfrak{g}(\Gamma)$. The first main result of this paper is described as follows.

Theorem 1.1. Let $Q$ be a quiver without loops and with an admissible action of a finite abelian subgroup $G \subseteq \operatorname{Aut}(\mathbb{k} Q)$, and $\mathbb{k}$ be an algebraically closed field with chark $\nmid|G|$. Assume that $\Gamma$ and $\widehat{Q}$ are the valued graph and generalized $M c K a y$ quiver associated to $(Q, G)$, respectively. Then

(1) the images under $h$ of the dimension vectors of all the indecomposable $\widehat{Q}$-representations give the positive root system of the Kac-Moody algebra $\mathfrak{g}(\Gamma)$; 
(2) for each positive real root $\alpha$ of $\mathfrak{g}(\Gamma)$, let $X$ be a $\widehat{Q}$-representation such that $h(\operatorname{dim} X)=\alpha$. Then there are $\left|G_{X}\right|$ indecomposable $\widehat{Q}$-representations (up to isomorphism) such that their dimension vectors under $h$ are $\alpha$.

The proof of this theorem is based on understanding the relationship among indecomposable $\widehat{Q}$-representations, indecomposable $\mathbb{k} Q * G$-modules and indecomposable $G$-invariant $Q$-representations. In the proof, we also need the dual between $(Q, G)$ and $(\widehat{Q}, G)$. This duality is first discussed in [18] for a finite quiver with an automorphism. Here we give a general and strict proof by the generalized McKay quiver.

Next we consider the relationship between Kac-Moody algebra $\mathfrak{g}(\Gamma)$ and the fixed point subalgebra $\mathfrak{g}^{\bar{G}}$ whenever char $\mathbb{k}=0$. The action of $G$ on $\widehat{Q}$ naturally induces an action on the derived algebra $\mathfrak{g}^{\prime}$ of $\mathfrak{g}$. Let $\Omega=\left\{g_{1}, g_{2}, \ldots, g_{n}\right\}$ be a set of generators of $G$. Following from [14], we lift $G$ to $\bar{G} \subseteq \operatorname{Aut}(\mathfrak{g})$ corresponding to a family of linear maps $\left\{\psi_{i}:=\psi_{g_{i}}: \mathfrak{h} / \mathfrak{h}^{\prime} \rightarrow \mathfrak{c} \mid g_{i} \in \Omega\right\}$, where $\mathfrak{c}$ is the center of $\mathfrak{g}, \mathfrak{h}$ and $\mathfrak{h}^{\prime}$ are the Cartan subalgebra of $\mathfrak{g}$ and $\mathfrak{g}^{\prime}$ respectively. Denote by $C$ the symmetrisable generalized Cartan matrix of the valued graph $\Gamma$. Then, we can give a realization $\left(\mathcal{H}^{\bar{G}},\left\{\epsilon_{i}\right\},\left\{h_{i}\right\}\right)$ of $C$ by the fixed point set $\mathfrak{h}^{\bar{G}}$ of $\mathfrak{h}$, and we obtain that:

Theorem 1.2. For the lifting $\bar{G}$ of $G$ corresponding to $\left\{\psi_{i}: \mathfrak{h} / \mathfrak{h}^{\prime} \rightarrow \mathfrak{c} \mid g_{i} \in \Omega\right\}$ such that $\psi_{i}\left(\left(\mathcal{H}+\mathfrak{h}^{\prime}\right) / \mathfrak{h}^{\prime}\right)=0$, there is a monomorphism

$$
\mathfrak{g}(\Gamma) \rightarrow \mathfrak{g}^{\bar{G}} \text {. }
$$

Moreover this monomorphism endows $\mathfrak{g}^{\bar{G}}$ with an integrable $\mathfrak{g}(\Gamma)$-module structure under the adjoint action of $\mathfrak{g}(\Gamma)$. In particular, if $Q$ is a finite union of Dynkin quivers, then $\mathfrak{g}(\Gamma) \cong \mathfrak{g}^{\bar{G}}$ as Lie algebras.

In the end of this paper, two examples are given to elucidate our results.

Throughout this paper, let $\mathbb{k}$ denote an algebraic closed field and $\mathbb{Z}$ denote the set of integers. We denote by $G$ the finite group such that chark $\nmid|G|$, denote by mod- $\Lambda$ the category of (left) $\Lambda$-modules for any $\mathbb{k}$-algebra $\Lambda$.

\section{Preliminaries}

2.1. Recall that a quiver $Q=(I, E)$ is an oriented graph with $I$ the set of vertices and $E$ the set of arrows. A quiver $Q$ is said to be finite if $I$ and $E$ are all finite set. An arrow in $Q$ is called a loop if its staring vertex coincides with its terminating vertex. In this paper we only consider a finite quiver without loops. Therefore we have a path algebra $\mathbb{k} Q$ for a quiver $Q$ (see $[1,3]$ ).

A representation $X=\left(X_{i}, X_{\alpha}\right)$ of the quiver $Q=(I, E)$ consists of a family of $\mathbb{k}_{k}$-vector spaces $X_{i}$ for $i \in I$, together with a family of $\mathbb{k}$-linear maps $X_{\alpha}$ : $X_{i} \rightarrow X_{j}$ for $\alpha: i \rightarrow j$ in $E$. Given two representations $X$ and $Y$ of $Q$, a morphism $\varphi: X \rightarrow Y$ is given by a family of $\mathbb{k}$-linear maps $\varphi_{i}: X_{i} \rightarrow Y_{i}(i \in I)$ such that $\varphi_{j} \circ X_{\alpha}=Y_{\alpha} \circ \varphi_{i}$ for each arrow $\alpha: i \rightarrow j$. It is well-known that 
the category of representations of $Q$ is naturally equivalent to the category of $\mathbb{k}_{\mathrm{k}} Q$-modules (see $\left.[1,3]\right)$. Thus we always identify a $\mathbb{k}_{\mathrm{k}} Q$-module $X$ with a $Q$-representation $\left(X_{i}, X_{\alpha}\right)$ in this paper.

2.2. Assume that $\Lambda$ is a $\mathbb{k}$-algebra and $G$ acts on $\Lambda$, the skew group algebra of $\Lambda$ under the action of $G$ is by definition the $\mathbb{k}$-algebra whose underlying $\mathbb{k}$-vector space is $\Lambda \otimes_{\mathbb{k}} \mathbb{k}[G]$ and whose multiplication is defined by

$$
(\lambda \otimes g)\left(\lambda^{\prime} \otimes g^{\prime}\right)=\lambda g\left(\lambda^{\prime}\right) \otimes g g^{\prime}
$$

for all $\lambda, \lambda^{\prime} \in \Lambda$ and $g, g^{\prime} \in G$ (see [18]). For convenience, we denote this algebra by $\Lambda * G$, denote the element $\lambda \otimes g$ in $\Lambda * G$ by $\lambda g$. Note that $\Lambda$ and $\mathbb{k}[G]$ can be viewed as subalgebras of $\Lambda * G$.

Let $\Lambda=\mathbb{k} Q$ be the path algebra for a quiver $Q=(I, E)$. Assume that the cation of $G$ on $\mathbb{k} Q$ permutes the set of primitive idempotents $\left\{e_{i} \mid i \in I\right\}$ and stabilizes the vector space spanned by the arrows. Let $\mathcal{I}$ denote a set of representatives of the orbits of $I$ under the action of $G$. For any $i \in I$, let $G_{i}$ denote the subgroup of $G$ stabilizing $e_{i}$. For each $i \in I$, there exist some $g \in G$ such that $g^{-1}(i) \in \mathcal{I}$. We fix such a $g$ and denote it by $\kappa_{i}$. Let $\mathcal{O}_{i}$ be the orbit of $i$ under the action of $G$. For $(i, j) \in \mathcal{I}^{2}, G$ acts on $\mathcal{O}_{i} \times \mathcal{O}_{j}$ by the diagonal action. A set of representatives of the orbits of this action will be denoted by $\mathcal{F}_{i j}$.

For $i, j \in I$, we denote by $E_{i j} \subseteq \mathbb{k} Q$ the vector space spanned by the arrows from $i$ to $j$ and regard it as a left and right $\mathbb{k}\left[G_{i j}\right]:=\mathbb{k}\left[G_{i} \cap G_{j}\right]$-module by restricting the action of $G$. In [5] Demonet defined the quiver $\widehat{Q}=(\widehat{I}, \widehat{E})$ as follows

$$
\widehat{I}=\bigcup_{i \in \mathcal{I}}\{i\} \times \operatorname{irr} G_{i},
$$

where $\operatorname{irr} G_{i}$ is a set of representatives of isomorphism classes of irreducible representations of $G_{i}$. The set of arrows of $\widehat{Q}$ from $(i, \rho)$ to $(j, \sigma)$ is a basis of

$$
\bigoplus_{\left(i^{\prime}, j^{\prime}\right) \in \mathcal{F}_{i j}} \operatorname{Hom}_{\mathbb{k}\left[G_{i^{\prime} j^{\prime}}\right]}\left(\left.\left(\rho \cdot \kappa_{i^{\prime}}\right)\right|_{G_{i^{\prime} j^{\prime}}},\left.\left(\sigma \cdot \kappa_{j^{\prime}}\right)\right|_{G_{i^{\prime} j^{\prime}}} \otimes_{\mathbb{k}} E_{i^{\prime} j^{\prime}}\right),
$$

where the representation $\rho \cdot \kappa_{i^{\prime}}$ of $G_{i^{\prime}}$ is the same as $\rho$ as a $\mathbb{k}$-vector space, and $\left(\rho \cdot \kappa_{i^{\prime}}\right) g=\rho \kappa_{i^{\prime}} g \kappa_{i^{\prime}}^{-1}$ for each $g \in G_{i^{\prime}}=\kappa_{i^{\prime}}^{-1} G_{i} \kappa_{i^{\prime}}$. Demonet yielded the following theorem.

Theorem 2.1 (see [5]). The category $\bmod -\mathbb{k} \widehat{Q}$ is equivalent to the category $\bmod -\mathbb{k} Q * G$.

In particular, if the quiver $Q$ is a single vertex with $m$ loops, we can view $G$ as a subgroup of $\mathbf{G L}_{m}(\mathbb{k})$. Then the quiver $\widehat{Q}$ is just the McKay quiver of $G$. Thus, we view $\widehat{Q}$ as a generalization of McKay quiver in general. Furthermore, for any factor algebra $\mathbb{k}_{Q} / J$, the shew group algebra $(\mathbb{k} Q / J) * G$ is Morita equivalent to a factor algebra of $\mathbb{k} \widehat{Q}$. This implies that the generalized McKay quiver can realize the Garbiel quiver of $\Lambda * G$ for any basic algebra $\Lambda$. 
2.3. For a quiver $Q=(I, E)$, there is a corresponding symmetric generalized Cartan matrix $A=\left(a_{i j}\right)$ indexed by $I$ with entries

$$
a_{i j}= \begin{cases}2, & i=j \\ -\mid\{\text { edges between vertices } i \text { and } j\} \mid, & i \neq j .\end{cases}
$$

It is obvious that $A$ is independent of the orientation of $Q$.

Denote by $\mathfrak{g}(Q)$ the associated symmetric Kac-Moody algebra corresponding to $A$ with the simple root set $\Pi=\left\{\varepsilon_{i} \mid i \in I\right\}$ and root system $\Delta_{Q}$. The root lattice $\mathbb{Z} I$ of $Q$ is the free abelian group on $\Pi$, with the partially order such that $\alpha=\sum_{i \in I} \alpha_{i} \varepsilon_{i} \geq 0$ if and only if $\alpha_{i} \geq 0$ for all $i \in I$. We endow $\mathbb{Z} I$ with a symmetric bilinear form $(-,-)_{Q}$ via $\left(\varepsilon_{i}, \varepsilon_{j}\right)_{Q}=a_{i j}$. Then, for each vertex $i \in I$, we have a reflection $r_{i}: \alpha \mapsto \alpha-\left(\alpha, \varepsilon_{i}\right)_{Q} \varepsilon_{i}$. These reflections generate the Weyl group $\mathcal{W}(Q)$ of $Q$. The real roots of $Q$ are given by the images under $\mathcal{W}(Q)$ of the simple roots $\varepsilon_{i}$ and the imaginary roots are given by \pm the images under $\mathcal{W}(Q)$ of the fundamental set

$F_{Q}:=\left\{\alpha>0 \mid\left(\alpha, \varepsilon_{i}\right)_{Q} \leq 0\right.$ for all $i$ and the support of $\alpha$ is connected $\}$.

Suppose that the action of $G$ on path algebra $\mathbb{k} Q$ permutes the set of primitive idempotents. The action of $G$ is said to be admissible if no arrow connects to vertices in the same $G$-orbit. For any quiver $Q$ with an admissible action of $G$, we can construct a symmetric matrix $B=\left(b_{i j}\right)$ indexed by $\mathcal{I}$, where

$$
b_{i j}= \begin{cases}2\left|\mathcal{O}_{i}\right|, & i=j \\ -\mid\left\{\text { edges between vertices in } \mathcal{O}_{i} \text { and } \mathcal{O}_{j}\right\} \mid, & i \neq j\end{cases}
$$

Let $d_{i}:=b_{i i} / 2=\left|\mathcal{O}_{i}\right|$ and $D=\operatorname{diag}\left(d_{i}\right)$. Then $C=\left(c_{i j}\right)=D^{-1} B$ is a symmetrisable generalized Cartan matrix indexed by $\mathcal{I}$. It is well-known that there is a unique valued graph $\Gamma$ corresponding to the matrix $C$ by [7]. The valued graph $\Gamma$ has the vertex set $\mathcal{I}$ and an edge $i-j$ equipped with the ordered pair $\left(\left|c_{j i}\right|,\left|c_{i j}\right|\right)$ whenever $c_{i j} \neq 0$. Since the action of $G$ is admissible, $\Gamma$ has no loops. For each connected component $\Gamma^{\prime}$ of the graph $\Gamma$, we always take the representative set $\mathcal{I}$ such that the underlying graph of the full subquiver generated by the vertices in $\Gamma^{\prime}$ is connected.

Denote by $\mathfrak{g}(\Gamma)$ for the associated symmetric Kac-Moody algebra corresponding to $C$. The simple root set and root system of $\Gamma$ are denoted by $\Pi_{\Gamma}=\left\{\bar{\varepsilon}_{i} \mid i \in \mathcal{I}\right\}$ and $\Delta_{\Gamma}$. Let $\mathbb{Z} \mathcal{I}$ denote the root lattice of $\Gamma$. There is a symmetric bilinear form $(-,-)_{\Gamma}$ determined by $B$ on $\mathbb{Z I}$ such that $\left(\bar{\varepsilon}_{i}, \bar{\varepsilon}_{j}\right)_{\Gamma}=b_{i j}$, and a reflection $\gamma_{i}$ on $\mathbb{Z I}$ defined by

$$
\gamma_{i}: \alpha \mapsto \alpha-\frac{1}{d_{i}}\left(\alpha, \bar{\varepsilon}_{i}\right)_{\Gamma} \bar{\varepsilon}_{i}
$$

for each $i \in \mathcal{I}$. These reflections generate the Weyl group $\mathcal{W}(\Gamma)$ of $\Gamma$. Similarly, we have the real roots and the imaginary roots associated to $\Gamma$ (see [13]). 


\section{Proof of Theorem 1.1}

From now on, unless otherwise stated we fix a finite group $G \subseteq \operatorname{Aut}\left(\mathbb{k}_{k} Q\right)$ and assume that the action of $G$ is admissible. Let $\widehat{Q}$ and $\Gamma$ be the generalized Mckay quiver and the valued graph corresponding to $(Q, G)$. In this section, we show that the correspondence between indecomposable representations of $\widehat{Q}$ and the positive root system of $\Gamma$.

3.1. The group $G$ acts naturally on the root lattice $\mathbb{Z} I$, i.e., $g\left(\varepsilon_{i}\right)=\varepsilon_{g(i)}$ for any $g \in G$. It is easy to check that this action preserves the partial order $\geq$ and the bilinear form $(-,-)_{Q}$ is $G$-invariant. Let

$$
(\mathbb{Z} I)^{G}:=\{\alpha \in \mathbb{Z} I \mid g(\alpha)=\alpha \text { for any } g \in G\} .
$$

There is a canonical bijection

$$
f: \quad(\mathbb{Z} I)^{G} \longrightarrow \mathbb{Z I}
$$

given by

$$
f\left(\sum_{i \in I} \alpha_{i} \varepsilon_{i}\right)=\sum_{i \in \mathcal{I}} \alpha_{i} \bar{\varepsilon}_{i}
$$

The admissibility of the action of $G$ implies that the reflections $r_{i}$ and $r_{j}$ commute whenever $i$ and $j$ lie in the same $G$-orbit. Therefore the element

$$
S_{i}:=\prod_{i^{\prime} \in \mathcal{O}_{i}} r_{i^{\prime}} \in \mathcal{W}(Q)
$$

is well-defined for any $i \in \mathcal{I}$. Note that $g \circ r_{i}=r_{g(i)} \circ g$ for any $g \in G$, we have $S_{i} \in C_{G}(\mathcal{W}(Q))$, the set of elements in the Weyl group commuting with the action of $G$. By induction on the length of the element in $C_{G}(\mathcal{W}(Q))$, it is easy to check that $C_{G}(\mathcal{W}(Q))$ is generated by $S_{i}, i \in \mathcal{I}$.

Similar to [12, Lemma 3], we have:

Lemma 3.1. For any $\alpha, \beta \in(\mathbb{Z} I)^{G}$, we have

(1) $(\alpha, \beta)_{Q}=(f(\alpha), f(\beta))_{\Gamma}$.

(2) $f\left(S_{i}(\alpha)\right)=\gamma_{i}(f(\alpha)) \in \mathbb{Z} \mathcal{I}$ for $i \in \mathcal{I}$.

(3) The map $\gamma_{i} \mapsto S_{i}$ induces a group isomorphism $\mathcal{W}(\Gamma) \stackrel{\simeq}{\longrightarrow} C_{G}(\mathcal{W}(Q))$.

Proof. (1) Set $\varepsilon^{i}:=\sum_{i^{\prime} \in \mathcal{O}_{i}} \varepsilon_{i^{\prime}}$. Then $\left\{\varepsilon^{i} \mid i \in \mathcal{I}\right\}$ is a basis of $(\mathbb{Z} I)^{G}$. Since

$$
\left(\varepsilon^{i}, \varepsilon^{j}\right)_{Q}=\sum_{\substack{i^{\prime} \in \mathcal{O}_{i} \\ j^{\prime} \in \mathcal{O}_{j}}} a_{i^{\prime} j^{\prime}}=b_{i j}=\left(\bar{\varepsilon}_{i}, \bar{\varepsilon}_{j}\right)_{\Gamma}
$$

for any $i, j \in \mathcal{I},(1)$ is obvious.

(2) Since the bilinear form $(-,-)_{Q}$ is $G$-invariant, we have

$$
S_{i}(\alpha)=\alpha-\sum_{i^{\prime} \in \mathcal{O}_{i}}\left(\alpha, \varepsilon_{i^{\prime}}\right)_{Q} \varepsilon_{i^{\prime}}=\alpha-\sum_{i^{\prime} \in \mathcal{O}_{i}} \frac{1}{d_{i}}\left(\alpha, \sum_{j \in \mathcal{O}_{i}} \varepsilon_{j}\right)_{Q} \varepsilon_{i^{\prime}}=\alpha-\frac{1}{d_{i}}\left(f(\alpha), \bar{\varepsilon}_{i^{\prime}}\right)_{\Gamma} \varepsilon^{i}
$$


by (1). We obtain that

$$
f\left(S_{i}(\alpha)\right)=f(\alpha)-\frac{1}{d_{i}}\left(f(\alpha), \bar{\varepsilon}_{i^{\prime}}\right)_{\Gamma} \bar{\varepsilon}_{i}=\gamma_{i}(f(\alpha)) .
$$

(3) By the result of (2), it is easy to check that $\gamma_{i}$ and $S_{i}$ satisfy the same relations. Thus $\mathcal{W}(\Gamma) \cong C_{G}(\mathcal{W}(Q))$.

For a given $\alpha \in \mathbb{Z} I$, let $H_{\alpha}=\{g \in G \mid g(\alpha)=\alpha\}$. Then $H_{\alpha}$ is a subgroup of $G$. We denote by $G_{\alpha}$ a complete set of representatives of left cosets of $H_{\alpha}$ in $G$, and let

$$
\Sigma(\alpha):=\sum_{g \in G_{\alpha}} g(\alpha)
$$

Obviously, $\Sigma(\alpha) \in(\mathbb{Z} I)^{G}$ and we have:

Lemma 3.2. The map $\alpha \mapsto f(\Sigma(\alpha))$ induces a surjection $\pi: \Delta_{Q} \rightarrow \Delta_{\Gamma}$. Moreover, if $f(\Sigma(\alpha))$ is a real root, $\alpha$ has to be real and unique up to $G$-orbit.

Proof. First, for any $\omega \in C_{G}(\mathcal{W}(Q))$, we have $H_{\alpha}=H_{\omega(\alpha)}$ since the action of $C_{G}(\mathcal{W}(Q))$ and the action of $G$ on $\mathbb{Z} I$ is commutative. Thus we can take $G_{\alpha}=G_{\omega(\alpha)}$ for any $\omega \in C_{G}(\mathcal{W}(Q))$.

We now consider $\beta:=\omega^{\prime}(f(\Sigma(\alpha)))$ with $\omega^{\prime} \in \mathcal{W}(\Gamma)$. Let $\omega \in C_{G}(\mathcal{W}(Q))$ be the element corresponding to $\omega^{\prime}$ under the isomorphism in Lemma 3.1(3). Then $\beta=f(\omega(\Sigma(\alpha)))=f(\Sigma(\omega(\alpha)))$ has connected support since the support of $\alpha$ is connected. It is either positive or negative since $\Sigma$ preserves the partial order $\geq$. Denote by $\mathcal{O}_{\beta}$ the orbit of $\beta$ under the action of $\mathcal{W}(\Gamma)$. Then

- if all elements in $\mathcal{O}_{\beta}$ are positive, the element in $\mathcal{O}_{\beta}$ with minimal height lies in $F_{\Gamma}$;

- if all elements in $\mathcal{O}_{\beta}$ are negative, the element in $\mathcal{O}_{\beta}$ with maximal height lies in $-F_{\Gamma}$;

- otherwise, there exists a positive number $m$ and $i \in \mathcal{I}$ such that $m \bar{\varepsilon}_{i} \in$ $\mathcal{O}_{\beta}$.

In the last case, we have $\omega(\alpha)=m \varepsilon_{i^{\prime}}$ for some $\omega \in \mathcal{W}(Q), i^{\prime} \in \mathcal{O}_{i}$. But $\omega(\alpha) \in \Delta_{Q}$, we must have $m=1$ and so that $\bar{\varepsilon}_{i} \in \mathcal{O}_{\beta}$. Thus $\beta$ is a root of $\Gamma$ and $\pi: \Delta_{Q} \rightarrow \Delta_{\Gamma}, \alpha \mapsto f(\Sigma(\alpha))$ is well-defined.

Now, we prove that the map $\pi$ is surjective. Here we only need to show that $F_{\Gamma}$ lies in the image of $\pi$. For any $\beta \in F_{\Gamma}, \gamma:=f^{-1}(\beta)$ satisfies

$$
0 \geq\left(\beta, \bar{\varepsilon}_{i}\right)_{\Gamma}=\left(\gamma, \Sigma\left(\varepsilon_{i^{\prime}}\right)\right)_{Q}=\sum_{g \in G_{\varepsilon_{i^{\prime}}}}\left(\gamma, g\left(\varepsilon_{i^{\prime}}\right)\right)_{Q}=d_{i}\left(\gamma, \varepsilon_{i^{\prime}}\right)_{Q}
$$

for any $i \in \mathcal{I}$ and $i^{\prime} \in \mathcal{O}_{i}$. Thus any connected component $\alpha$ of $\gamma$ lies in $F_{Q}$ and $\Sigma(\alpha)=\gamma$. By Lemma 3.1(3) we get the proof.

For any $g \in G$, we have an additive autoequivalence functor

$$
\begin{aligned}
F_{g}: \quad \bmod -\mathbb{k}_{k} Q & \longrightarrow \bmod -\mathbb{k}_{k} Q \\
M & \mapsto{ }^{g} M
\end{aligned}
$$


where the $\mathbb{k} Q$-module ${ }^{g} M$ is defined by taking the same underlying vector space as $M$ with the action $m \cdot \lambda=m g^{-1}(\lambda)$ for $m \in M$ and $\lambda \in \mathbb{k} Q$, and $F_{g}(\psi)=\psi$ for any homomorphism $\psi: M \rightarrow N$. Let $\left(M_{i}, M_{\alpha}\right)_{i \in I, \alpha \in E}$ be the $Q$-representation corresponding to $M$. Then the $Q$-representation ${ }^{g} M$ is $\left({ }^{g} X_{i},{ }^{g} X_{\alpha}\right)_{i \in I, \alpha \in E}$, where ${ }^{g} X_{i}=X_{g^{-1}(i)}$ and ${ }^{g} X_{\alpha}=\sum_{\beta} \zeta_{\beta} X_{\beta}$ if $g^{-1}(\alpha)=$ $\sum_{\beta} \zeta_{\beta} \beta, \beta \in E, \zeta_{\beta} \in \mathbb{k}$.

A $\mathbb{k} Q$-module $M$ is said to be $G$-invariant if $F_{g}(M) \cong M$ for any $g \in G$; a $G$-invariant $\mathbb{k}_{k} Q$-module $M$ is said to be indecomposable $G$-invariant if $M$ is non-zero and $M$ cannot be written as a direct sum of two non-zero $G$-invariant $\mathbb{k} Q$-modules. It is known that $\mathbb{k}_{k} Q$-module $M$ has a $\mathbb{k}_{\mathrm{k}} Q * G$-module structure if and only if $M$ is $G$-invariant, and the full subcategory of $\bmod -\mathbb{k} Q$ generated by the $G$-invariant $\mathbb{k}_{k} Q$-module is also a Krull-Schmidt category (see [10]).

For a given $\mathbb{k} Q$-module $M$, we let $H_{M}:=\left\{g \in G \mid F_{g}(M) \cong M\right\}$ and $G_{M}$ be a complete set of left coset representatives of $H_{M}$ in $G$. Then for each $\mathbb{k} Q$-module $M$, we define a $G$-invariant $\mathbb{k} Q$-module

$$
\sum(M):=\bigoplus_{g \in G_{M}}{ }^{g} M .
$$

It is easy to see that each $G$-invariant $\mathbb{k}_{k} Q$-module has this form. For each $\mathbb{k}_{k} Q$ module $M$, we denote the dimension vector of $M$ by the linear combination $\operatorname{dim} X:=\sum_{i \in I} \operatorname{dim} X_{i} \varepsilon_{i} \in \mathbb{Z} I$. It is easy to see that $\operatorname{dim} F_{g}(M)=g(\operatorname{dim} M)$ for any $g \in G$ and $M \in \bmod -\mathbb{k}_{k} Q$.

Proposition 3.3. For any indecomposable $G$-invariant $\mathbb{k}_{k} Q$-module $M$, we have $f(\operatorname{dim} M)$ is a root of $\Gamma$. Moreover, for any positive real root $\beta$ of $\Gamma$, there is a unique (up to isomorphism) indecomposable $G$-invariant $\mathbb{k} Q$-module $M$ with $\frac{1}{2}(\operatorname{dim} M, \operatorname{dim} M)_{Q}$ indecomposable summands (as $\mathbb{k} Q$-module) such that $f(\operatorname{dim} M)=\beta$.

Proof. Let $N$ be an indecomposable $\mathbb{k} Q$-module and $\alpha:=\operatorname{dim} N$. Then $\sum(N)$ is an indecomposable $G$-invariant $\mathbb{k}_{k} Q$-module with dimension vector $\sum_{g \in G_{N}} g(\alpha)$. We claim that

$$
\sum_{g \in G_{N}} g(\alpha)=m \Sigma(\alpha)
$$

for some positive integer $m$. Indeed, since $H_{N} \subseteq H_{\alpha}$, we have $\left|H_{\alpha}\right|=m\left|H_{N}\right|$ and so that $\left|G_{N}\right|=m\left|G_{\alpha}\right|$ for some positive integer $m$. Note that

$$
\sum_{g \in G_{\alpha}} g(\alpha)=\frac{\sum_{g \in G} g(\alpha)}{\left|H_{\alpha}\right|} \text { and } \sum_{g \in G_{N}} g(\alpha)=\frac{\sum_{g \in G} g(\alpha)}{\left|H_{N}\right|}
$$

we obtain that

$$
\operatorname{dim} \sum(N)=\sum_{g \in G_{N}} g(\alpha)=m \sum_{g \in G_{\alpha}} g(\alpha)=m \Sigma(\alpha) .
$$


In particular, if $\alpha$ is a real root of $Q$, then $H_{N}=H_{\alpha}$ and so that we take $G_{N}=G_{\alpha}$ in this case. Therefore, $f\left(\operatorname{dim} \sum(N)\right) \in \Delta_{\Gamma}$. Note that for every indecomposable $G$-invariant $\mathbb{k}_{k} Q$-module $M$, there is an indecomposable $\mathbb{k}_{k} Q$ module $N$ such that $M \cong \sum(N)$, we get $f(\operatorname{dim} M) \in \Delta_{\Gamma}$.

If $\beta:=f(\operatorname{dim} M)$ is a real root with $f(\operatorname{dim} M)=\omega^{\prime}\left(\bar{\varepsilon}_{i}\right)$ for some $\omega^{\prime} \in$ $\mathcal{W}(\Gamma)$ and $i \in \mathcal{I}$, then $\operatorname{dim} M=\omega\left(\Sigma\left(\varepsilon_{i^{\prime}}\right)\right)=\Sigma\left(\omega\left(\varepsilon_{i^{\prime}}\right)\right)$ for any $i^{\prime} \in \mathcal{O}_{i}$, where $\omega \in C_{G}(\mathcal{W}(Q))$ corresponding to $\omega^{\prime}$, by the proof of Lemma 3.2. Denote by $N$ the unique indecomposable $\mathbb{k} Q$-module with $\operatorname{dim} N=\omega\left(\varepsilon_{i^{\prime}}\right)$, then $M=\sum(N)$ is the unique indecomposable $G$-invariant $\mathbb{k} Q$-module satisfying $\operatorname{dim} M=\omega\left(\Sigma\left(\varepsilon_{i^{\prime}}\right)\right)$ and $M$ is independent on the taking of $i^{\prime} \in \mathcal{O}_{i}$. Finally, note that

$$
\frac{1}{2}(\operatorname{dim} M, \operatorname{dim} M)_{Q}=\frac{1}{2}\left(\Sigma\left(\varepsilon_{i^{\prime}}\right), \Sigma\left(\varepsilon_{i^{\prime}}\right)\right)_{Q}=d_{i}=\left|G_{\varepsilon_{i^{\prime}}}\right|=\left|G_{N}\right|,
$$

we are done.

We suppose now that $G$ is abelian with identity $\varpi$, and let

$$
e:=\sum_{i \in \mathcal{I}} e_{i} \varpi \in \mathbb{k} Q * G
$$

where $e_{i}$ is the idempotent element of $\mathbb{k}_{k} Q$ corresponding to vertex $i \in I$. By the proof of $\left[5\right.$, Theorem 1], we know that $\mathbb{k}_{k} Q * G$ is Morita equivalent to $e \mathbb{k}_{k} Q * G e$ and $e \mathbb{k} Q * G e \cong \mathbb{k} \widehat{Q}$. Thus we view the functor

$$
\begin{aligned}
E: \quad \bmod -\mathbb{k} Q * G & \longrightarrow \quad \bmod -\mathbb{k}_{\mathrm{Q}} \widehat{Q} \\
M & \mapsto e M
\end{aligned}
$$

as the equivalence functor between $\bmod -\mathbb{k} Q * G$ and $\bmod -\mathbb{k} \widehat{Q}$. Denote by

$$
\begin{array}{ll}
F:=\mathbb{k}_{k} Q * G \otimes_{\mathbb{k} Q}-: \quad \bmod -\mathbb{k}_{k} \longrightarrow \bmod -\mathbb{k}_{k} Q * G, \\
H:=\left.\operatorname{Res}\right|_{\mathbb{k} Q}: & \bmod -\mathbb{k}_{k} * G \longrightarrow \bmod -\mathbb{k}_{k} Q .
\end{array}
$$

Following from [5, Theorem 1.1], $(H, F)$ and $(F, H)$ are adjoint pairs.

Moreover, for any $\mathbb{k} \widehat{Q}$-module $X, H E^{-1}(X)$ is a $G$-invariant $\mathbb{k} Q$-module and there is a $\mathbb{k} Q$-module $M$ such that $H E^{-1}(X) \cong \sum(M)$, where $E^{-1}$ is the quasi-inverse of $E$. Identifying $X$ with a $\widehat{Q}$-representation $\left(X_{i \rho}, X_{\alpha}\right)$, we have

$$
\sum_{\rho \in \operatorname{irr} G_{i}} X_{i \rho} \cong e_{i} H E^{-1}(X) e_{i} \cong \bigoplus_{g \in G_{M}}\left({ }^{g} M\right)_{i} .
$$

Suppose $\operatorname{dim} X:=\sum_{(i \rho) \in \widehat{I}} \alpha_{i \rho} \varepsilon_{i \rho}$, then

$$
\sum_{\rho \in \operatorname{irr} G_{i}} \alpha_{i \rho}=\sum_{g \in G_{M}} \operatorname{dim}\left({ }^{g} M\right)_{i}=f\left(\operatorname{dim} \sum(M)\right)_{i} .
$$

Therefore, the Moriat equivalence and the restriction functor induce a map

$$
h: \mathbb{Z} \widehat{I} \longrightarrow \mathbb{Z I}
$$


given by $h(\alpha)_{i}=\sum_{\rho \in \operatorname{irr} G_{i}} \alpha_{i \rho} \bar{\varepsilon}_{i}$ for any $\alpha=\sum_{(i \rho) \in \widehat{I}} \alpha_{i \rho} \varepsilon_{i \rho} \in \mathbb{Z} \widehat{I}$. The restriction of $h$ to the root system $\Delta_{\widehat{Q}}$ is also denoted by $h$. Then $h: \Delta_{\widehat{Q}} \rightarrow \Delta_{\Gamma}$ is well-defined since $X$ is an indecomposable $\mathbb{k} \widehat{Q}$-module if and only if $M$ is an indecomposable $\mathbb{k}_{\mathrm{k}} Q$-module. By Proposition 3.3, we have:

Corollary 3.4. For any indecomposable $\widehat{Q}$-representation $X, h(\operatorname{dim} X)$ is a positive root of $\Gamma$.

Up to now, we have obtained the map $h: \mathbb{Z} \widehat{I} \rightarrow \mathbb{Z I}$ and have shown the half of Theorem 1.1(1). Before completing the proof of Theorem 1.1, we should define an action of $G$ on $\mathbb{k}_{k} \widehat{Q}$ and give the dual between $(Q, G)$ and $(\widehat{Q}, G)$. In the following subsection, we first describe the duality of $(Q, G)$.

3.2. We write the abelian group $G$ as the product of finite cyclic groups, i.e.,

$$
G=\left\langle g_{1}\right\rangle \times\left\langle g_{2}\right\rangle \times \cdots \times\left\langle g_{n}\right\rangle,
$$

where the order of $g_{i}$ is $m_{i}$ for $1 \leq i \leq n$. Then $|G|=m_{1} m_{2} \cdots m_{n}$.

We now define an action of $G$ on $\widehat{Q}$. Since $G$ is abelian, all the characters $\chi$ of $G$ are linear. The set of all the characters of $G$ is an abelian group with the multiplication

$$
\chi \chi^{\prime}(g)=\chi(g) \chi^{\prime}(g)
$$

for all $g \in G$. We denote this group by $\widetilde{G}$. Setting $\varphi: G \rightarrow \widetilde{G}$ by

$$
\varphi(g)=\chi_{g}, \quad \chi_{g}\left(g^{\prime}\right)=\xi_{1}^{t_{1} s_{1}} \xi_{2}^{t_{2} s_{2}} \cdots \xi_{n}^{t_{n} s_{n}}
$$

if $g=g_{1}^{t_{1}} g_{2}^{t_{2}} \cdots g_{n}^{t_{n}}$ and $g^{\prime}=g_{1}^{s_{1}} g_{2}^{s_{2}} \cdots g_{n}^{s_{n}}$, where $\xi_{i}$ is a primitive $m_{i}$-th root of unity. It is easy to see that $\varphi$ is a group isomorphism. By [18], we define a linear action of $G$ on $\mathbb{k} Q * G$ by setting

$$
g(\lambda h)=\chi_{g}(h) \lambda h
$$

for all $g \in G, \lambda h \in \mathbb{k}_{k} Q * G$. Then $G \subseteq \operatorname{Aut}(\mathbb{k} Q * G)$. By [18, Proposition 5.1], we have:

Proposition 3.5. The map $\psi:(\mathbb{k} Q * G) * G \rightarrow \operatorname{End}_{\mathbb{k} Q}\left(\mathbb{k}_{k} Q * G\right)$ defined by

$$
\psi(\lambda g h)\left(\mu h^{\prime}\right)=\chi_{h}\left(h^{\prime}\right) \lambda g \mu h^{\prime}
$$

is an algebra isomorphism. It follows that $(\mathbb{k} Q * G) * G$ is Morita equivalent to $\mathbb{k}_{k} Q$.

Moreover, note that $\chi_{g}(\varpi)=1$ for any $g \in G$, where $\varpi$ is the identity of $G$. We get $g(e)=e$. Denote by $\vartheta: e \mathbb{k} Q * G e \rightarrow \mathbb{k} \widehat{Q}$ the algebra isomorphism, then for any $a \in \mathbb{k} \widehat{Q}$, there is a unique $b \in e \mathbb{k} Q * G e$, such that $\vartheta(b)=a$. Let $g(a)=\vartheta(g(b))$ for any $g \in G$. This induces an action of $G$ on $\mathbb{k} \widehat{Q}$ such that $G \subseteq \operatorname{Aut}(\mathbb{k} \widehat{Q})$. Therefore, we get a skew group algebra $\mathbb{k} \widehat{Q} * G$ under this action. Let $\widehat{\widehat{Q}}$ be the generalized McKay quiver of $(\widehat{Q}, G)$. Then, there is a Morita equivalence between $\mathbb{k} \widehat{Q} * G$ and $\mathbb{k} \widehat{\widehat{Q}}$ by Theorem 2.1. Therefore, we have: 
Proposition 3.6. Let $\widehat{Q}$ be the generalized McKay quiver of $(Q, G)$ under the action of $G$ defined as above. Then the generalized McKay quiver $\widehat{\widehat{Q}}$ of $(\widehat{Q}, G)$ coincides with $Q$.

Thus we get the dual between $(Q, G)$ and $(\widehat{Q}, G)$. Now, for the relationship between quivers $Q$ and $\widehat{Q}$, and the action of $G$ on $\widehat{Q}$, we give some more description. Note that the stabilizer $G_{i}$ of $i \in I$ has the form

$$
G_{i}=\left\langle g_{1}^{d_{i_{1}}}\right\rangle \times\left\langle g_{2}^{d_{i_{2}}}\right\rangle \times \cdots \times\left\langle g_{n}^{d_{i_{n}}}\right\rangle,
$$

where

and so that

$$
\nu_{i_{j}}:=\left|\left\langle g_{j}^{d_{i_{j}}}\right\rangle\right|=\frac{m_{j}}{d_{i_{j}}}, \quad 1 \leq j \leq n
$$

We set

$$
d_{i}=\left|\mathcal{O}_{i}\right|=\frac{|G|}{\left|G_{i}\right|}=d_{i_{1}} \times d_{i_{2}} \times \cdots \times d_{i_{n}} .
$$

$$
\begin{aligned}
& e_{\left(i, s_{i_{1}}, s_{i_{2}}, \ldots, s_{i_{n}}\right)} \\
= & \frac{1}{\left|G_{i}\right|} \sum_{j_{1}=0}^{\nu_{i_{1}}-1} \sum_{j_{2}=0}^{\nu_{i_{2}}-1} \cdots \sum_{j_{n}=0}^{\nu_{i_{n}}-1} \xi_{1}^{d_{i_{1}} j_{1} s_{i_{1}}} \xi_{2}^{d_{i_{2}} j_{2} s_{i_{2}}} \cdots \xi_{n}^{d_{i_{n}} j_{n} s_{i_{n}}} g_{1}^{d_{i_{1}} j_{1}} g_{2}^{d_{i_{2}} j_{2}} \cdots g_{n}^{d_{i_{n}} j_{n}} .
\end{aligned}
$$

Then one can check that $\left\{e_{\left(i, s_{i_{1}}, s_{i_{2}}, \ldots, s_{i_{n}}\right)} \mid s_{i_{j}} \in \mathbb{Z} / \nu_{i_{j}} \mathbb{Z}\right.$ for all $\left.1 \leq j \leq n\right\}$ is a complete set of primitive orthogonal idempotents of $\mathbb{k}\left[G_{i}\right]$.

It is obvious that

$$
g_{j}\left(e_{\left(i, s_{i_{1}}, s_{i_{2}}, \ldots, s_{i_{n}}\right)}\right)=e_{\left(i, s_{i_{1}}, \ldots, s_{i_{j-1}}, s_{i_{j}}^{\prime}, s_{i_{j+1}}, \ldots, s_{i_{n}}\right)}
$$

for any $1 \leq j \leq n$, where $s_{i_{j}}^{\prime} \in \mathbb{Z} / \nu_{i_{j}} \mathbb{Z}$ and $s_{i_{j}}^{\prime} \equiv s_{i_{j}}+1 \bmod \nu_{i_{j}}$. Since for each idempotent $e_{\left(i, s_{i_{1}}, s_{i_{2}}, \ldots, s_{i_{n}}\right)}$, there is a unique corresponding one dimensional irreducible representation $\rho$ of $G_{i}$ defined by the group homomorphism $\phi_{\rho}$ : $G_{i} \rightarrow \mathbb{k}, g_{j}^{d_{i_{j}}} \mapsto \xi^{d_{i_{j}} s_{i_{j}}}$, for $1 \leq j \leq n$. Thus we can index the vertex set $\widehat{I}$ by some sequences $\left(i, s_{i_{1}}, s_{i_{2}}, \ldots, s_{i_{n}}\right)$, i.e.,

$$
\widehat{I}=\left\{\left(i, s_{i_{1}}, s_{i_{2}}, \ldots, s_{i_{n}}\right) \mid i \in \mathcal{I}, s_{i_{j}} \in \mathbb{Z} / \nu_{i_{j}} \mathbb{Z} \text { for all } 1 \leq j \leq n\right\} .
$$

Then the action of $G$ on $\widehat{I}$ is clear and so that the orbit of $(i, \rho) \in \widehat{I}$ has the form

$$
\left\{\left(i, s_{i_{1}}, s_{i_{2}}, \ldots, s_{i_{n}}\right) \mid s_{i_{j}} \in \mathbb{Z} / \nu_{i_{j}} \mathbb{Z} \text { for } 1 \leq j \leq n\right\}=\left\{(i, \rho) \mid \rho \in \operatorname{irr} G_{i}\right\}
$$

for some $i \in \mathcal{I}$. Furthermore, it is easy to see that if the action of $G$ on $\mathbb{k}_{k} Q$ is admissible, then so is on $\mathbb{k} \widehat{Q}$.

For any $i, j \in \mathcal{I}, i^{\prime} \in \mathcal{O}_{i}$ and $j^{\prime} \in \mathcal{O}_{j}$, we consider the group $G_{i j}:=G_{i} \cap G_{j}=$ $\left\langle g_{1}^{t_{1}}\right\rangle \times\left\langle g_{2}^{t_{2}}\right\rangle \times \cdots \times\left\langle g_{n}^{t_{n}}\right\rangle$, where $t_{l}$ is the least common multiple of $d_{i_{l}}$ and $d_{j_{l}}$ for $1 \leq l \leq n$. Note that the vector space $E_{i^{\prime} j^{\prime}}$ spanned by arrows $\alpha: i^{\prime} \rightarrow j^{\prime}$ in $Q$ is a $\mathbb{k}\left[G_{i j}\right]$-bimodule, we can find a basis of $E_{i^{\prime} j^{\prime}}$ such that the action of $G_{i j}$ 
is diagonal. That is, if $g=g_{1}^{t_{1}} g_{2}^{t_{2}} \cdots g_{n}^{t_{n}} \in \mathbb{k}\left[G_{i j}\right]$, then for any basis element $\alpha^{\prime} \in E_{i^{\prime} j^{\prime}}$,

$$
g\left(\alpha^{\prime}\right)=\xi_{1}^{t_{1} r_{1}} \xi_{2}^{t_{2} r_{2}} \cdots \xi_{n}^{t_{n} r_{n}} \alpha^{\prime}
$$

for some $r_{1}, r_{2}, \ldots, r_{n} \in \mathbb{Z}$. Since $G_{i j}$ is abelian, the number of the basis elements of $E_{i^{\prime} j^{\prime}}$ is just the number of arrows from $i^{\prime}$ to $j^{\prime}$ in $Q$. Moreover, it is easy to see that the $t_{1} t_{2} \cdots t_{n}$ elements

$$
\alpha^{\prime}, g_{1}\left(\alpha^{\prime}\right), \ldots, g_{n}\left(\alpha^{\prime}\right), g_{1}^{2}\left(\alpha^{\prime}\right), g_{1} g_{2}\left(\alpha^{\prime}\right), \ldots, g_{1}^{t_{1}-1} g_{2}^{t_{2}-1} \cdots g_{n}^{t_{n}-1}\left(\alpha^{\prime}\right)
$$

are linearly independent. That is, for any arrow $\alpha: i^{\prime} \rightarrow j^{\prime}$ in $Q$, there are $t_{1} t_{2} \cdots t_{n}$ arrows in its orbit.

On the other hand, we can calculate that

$$
\begin{aligned}
& e_{\left(j, s_{j_{1}}, s_{j_{2}}, \ldots, s_{j_{n}}\right)} \alpha^{\prime} e_{\left(i, s_{i_{1}}, s_{i_{2}}, \ldots, s_{i_{n}}\right)} \\
& =\frac{d_{i} d_{j}}{|G|^{2}} \sum_{p_{1}=0}^{\nu_{i_{1}}-1} \cdots \sum_{p_{n}=0}^{\nu_{i_{n}}-1} \sum_{q_{1}=0}^{\nu_{j_{1}}-1} \cdots \sum_{q_{n}=0}^{\nu_{j_{n}}-1} \xi_{1}^{d_{i_{1}} p_{1} s_{i_{1}}+d_{j_{1}} q_{1} s_{j_{1}}} \cdots \xi_{n}^{d_{i_{n}} p_{n} s_{i_{n}}+d_{j_{n}} q_{n} s_{j_{n}}} \\
& g_{1}^{d_{j_{1}} q_{1}} \cdots g_{n}^{d_{j_{n}} q_{n}}\left(\alpha^{\prime}\right) g_{1}^{d_{i_{1}} p_{1}+d_{j_{1}} q_{1}} \cdots g_{n}^{d_{i_{n}} p_{n}+d_{j_{n}} q_{n}} \text {. }
\end{aligned}
$$

We write

$$
\begin{aligned}
& d_{i_{l}} p_{l}=P_{l} t_{l}+d_{i_{l}} p_{l}^{\prime}, \quad \text { where } 0 \leq P_{l}<\frac{m_{l}}{t_{l}}, \quad 0 \leq p_{l}^{\prime}<\frac{t_{l}}{d_{i_{l}}}, \\
& d_{j_{l}} q_{l}=P_{l}^{\prime} t_{l}+d_{j_{l}} q_{l}^{\prime}, \quad \text { where } 0 \leq P_{l}^{\prime}<\frac{m_{l}}{t_{l}}, \quad 0 \leq q_{l}^{\prime}<\frac{t_{l}}{d_{j_{l}}}, \\
& d_{i_{l}} k_{l} \equiv\left(P_{l}+P_{l}^{\prime}\right) t_{l}+d_{i_{l}} p_{l}^{\prime} \quad \bmod m_{l}, \quad \text { where } 0 \leq k_{l}<\nu_{i_{l}},
\end{aligned}
$$

for all $0 \leq l \leq n$. Then the right side of the equation becomes

$$
\begin{aligned}
\frac{d_{i} d_{j}}{|G|^{2}}\left(\sum_{P_{1}^{\prime}=0}^{\frac{m_{1}}{t_{1}}-1} \xi_{1}^{P_{1}^{\prime} t_{1}\left(r_{1}+s_{j_{1}}-s_{i_{1}}\right)}\right) \cdots\left(\sum_{P_{n}^{\prime}=0}^{\frac{m_{n}}{t_{n}}-1} \xi_{n}^{P_{n}^{\prime} t_{n}\left(r_{n}+s_{j_{n}}-s_{i_{n}}\right)}\right) \\
\left(\sum_{k_{1}=0}^{\nu_{i_{1}}-1} \cdots \sum_{k_{n}=0}^{\nu_{i_{n}}-1} \sum_{q_{1}^{\prime}=0}^{\frac{t_{1}}{d_{j_{1}}}-1} \cdots \sum_{q_{n}^{\prime}=0}^{\frac{t_{n}}{d_{j_{n}}}-1} \xi_{1}^{d_{i_{1}} k_{1} s_{i_{1}}+d_{j_{1}} q_{1}^{\prime} s_{j_{1}}} \cdots \xi_{n}^{d_{i_{n}} k_{n} s_{i_{n}}+d_{j_{n}} q_{n}^{\prime} s_{j_{n}}}\right. \\
\left.g_{1}^{d_{j_{1}} q_{1}^{\prime}} \cdots g_{n}^{d_{j_{n}} q_{n}^{\prime}}\left(\alpha^{\prime}\right) g_{1}^{d_{i_{1}} k_{1}+d_{j_{1}} q_{1}^{\prime}} \cdots g_{n}^{d_{i_{n}} k_{n}+d_{j_{n}} q_{n}^{\prime}}\right) .
\end{aligned}
$$

Note that

$$
\begin{aligned}
\left\{g_{1}^{d_{j_{1}} q_{1}^{\prime}} \cdots g_{n}^{d_{j_{n}} q_{n}^{\prime}}\left(\alpha^{\prime}\right) g_{1}^{d_{i_{1}} k_{1}+d_{j_{1}} q_{1}^{\prime}} \cdots g_{n}^{d_{i_{n}} k_{n}+d_{j_{n}} q_{n}^{\prime}}\right. & \\
& \left.\mid 0 \leq k_{l}<\nu_{i_{l}}, 0 \leq q_{l}^{\prime}<\frac{t_{l}}{d_{j_{l}}} \text { for } 1 \leq l \leq n\right\}
\end{aligned}
$$


is a linearly independent set. We obtain that $e_{\left(j, s_{j_{1}}, s_{j_{2}}, \ldots, s_{j_{n}}\right)} \alpha^{\prime} e_{\left(i, s_{i_{1}}, s_{i_{2}}, \ldots, s_{i_{n}}\right)} \neq$ 0 if and only if $s_{i_{l}} \equiv s_{j_{l}}+r_{l} \bmod \frac{m_{l}}{t_{l}}$ for all $0 \leq l \leq n$. It follows that there are $\frac{t_{1} \cdots t_{n}|G|}{d_{i} d_{j}}$ arrows in $\widehat{Q}$ for each arrow $\alpha: i^{\prime} \rightarrow j^{\prime}$ in $Q$.

Denote by $\widehat{A}=\left(a_{(i \rho)(j \sigma)}\right)_{\widehat{I} \times \widehat{I}}$ the Cartan matrix of $\widehat{Q}$, by $\widehat{\Gamma}$ the valued quiver corresponding to $(\widehat{Q}, G)$ and by $\widehat{C}=\left(\widehat{c}_{i j}\right)_{\mathcal{I} \times \mathcal{I}}=\widehat{D}^{-1} \widehat{B}$ the generalized Cartan matrix of $\widehat{\Gamma}$, where $\widehat{B}=\left(\widehat{b}_{i j}\right)_{\mathcal{I} \times \mathcal{I}}$ is symmetric, $\widehat{D}=\operatorname{diag}\left(\widehat{d}_{i}\right)$ is diagonal. Then

$$
\frac{1}{t_{1} \cdots t_{n}} \sum_{\substack{i^{\prime} \in \mathcal{O}_{i} \\ j^{\prime} \in \mathcal{O}_{j}}} a_{i^{\prime} j^{\prime}}=\frac{d_{i} d_{j}}{t_{1} \cdots t_{n}|G|} \sum_{\substack{\rho \in \operatorname{irr} G_{i} \\ \sigma \in \operatorname{irr} G_{j}}} a_{(i \rho)(j \sigma)} .
$$

It follows that $\widehat{b}_{i j}=\frac{|G|}{d_{i} d_{j}} b_{i j}, \widehat{D}=|G| D^{-1}, \widehat{B}=|G| D^{-1} B D^{-1}$ and $\widehat{C}=$ $(\widehat{D})^{-1} \widehat{B}=B D^{-1}=C^{T}$, the transpose of $C$. Therefore $\Gamma$ and $\widehat{\Gamma}$ are dual valued graphs in the sense of [13].

Remark 3.7. If $G \subseteq \operatorname{Aut}(\mathbb{k} Q)$ is a finite abelian group, we have given the dual of $(Q, G)$ and $(\widehat{Q}, G)$ (see Proposition 3.6). However, for a non-abelian group $G \subseteq \operatorname{Aut}\left(\mathbb{k}_{\mathrm{k}} Q\right)$, the conclusion does not hold in general. For example, let $Q$ be the quiver:

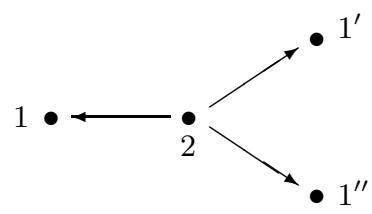

It is well-known that the quiver automorphism group of $Q$ is the group $S_{3}$. Accordingly, we obtain the generalized McKay quiver $\widehat{Q}$ of $\left(Q, S_{3}\right)$ as follows:

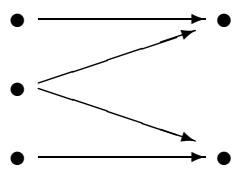

One can check that there does not exist a subgroup $G^{\prime}$ of $\operatorname{Aut}(\mathbb{k} \widehat{Q})$ such that the generalized McKay quiver of $\left(\widehat{Q}, G^{\prime}\right)$ is $Q$.

But if the action of $G$ is "good", there exists the duality still. For example, we consider the finite non-abelian group

$$
G=\left\langle a, b \mid a^{3}=b^{2}, b^{4}=1, a b a=b\right\rangle
$$


and the quiver $Q$ :

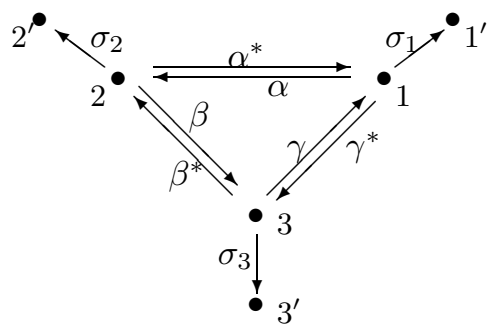

The action of $G$ is given by

\begin{tabular}{|c|c|c|c|c|c|c|c|c|}
\hline & $e_{1}$ & $e_{2}$ & $e_{3}$ & $e_{1^{\prime}}$ & $e_{2^{\prime}}$ & $e_{3^{\prime}}$ & $\alpha$ & $\alpha^{*}$ \\
\hline$a$ & $e_{2}$ & $e_{3}$ & $e_{1}$ & $e_{2^{\prime}}$ & $e_{3^{\prime}}$ & $e_{1^{\prime}}$ & $\beta$ & $\beta^{*}$ \\
\hline \multirow[t]{4}{*}{$b$} & $e_{1}$ & $e_{3}$ & $e_{2}$ & $e_{1^{\prime}}$ & $e_{2^{\prime}}$ & $e_{2^{\prime}}$ & $-\gamma^{*}$ & $\gamma$ \\
\hline & & $\beta$ & $\beta^{*}$ & $\gamma$ & $\gamma^{*}$ & $\sigma_{1}$ & $\sigma_{2}$ & \\
\hline & $a$ & $\gamma$ & $\gamma^{*}$ & $\alpha$ & $\alpha^{*}$ & $\sigma_{2}$ & $\sigma_{3}$ & \\
\hline & $b$ & $-\beta^{*}$ & $\beta$ & $-\alpha^{*}$ & $\alpha$ & $\sigma_{1}$ & $\sigma_{3}$ & \\
\hline
\end{tabular}

where $e_{i}$ is the idempotent element of $\mathbb{k}_{\mathrm{k}} Q$ corresponding to vertex $i, i \in$ $\left\{1,2,3,1^{\prime}, 2^{\prime}, 3^{\prime}\right\}$. By direct calculation, one see that the generalized McKay quiver of $(Q, G)$ is as follows:

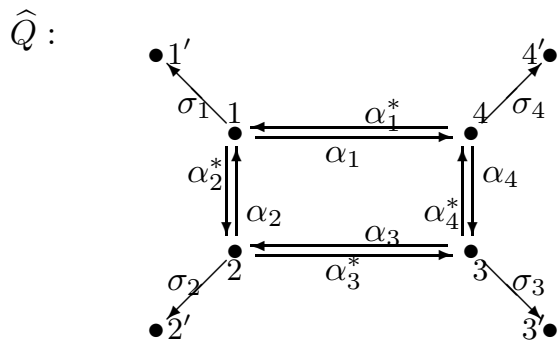

Now, we define an action of $G$ on $\mathbb{k} \widehat{Q}$ by setting

\begin{tabular}{c|cccccccccccc} 
& $e_{1}$ & $e_{2}$ & $e_{3}$ & $e_{4}$ & $e_{1^{\prime}}$ & $e_{2^{\prime}}$ & $e_{3^{\prime}}$ & $e_{4^{\prime}}$ & $\alpha_{1}$ & $\alpha_{2}$ & $\alpha_{3}$ & $\alpha_{4}$ \\
\hline$a$ & $e_{3}$ & $e_{4}$ & $e_{1}$ & $e_{2}$ & $e_{3^{\prime}}$ & $e_{4^{\prime}}$ & $e_{1^{\prime}}$ & $e_{4^{\prime}}$ & $\xi^{2} \alpha_{3}$ & $\xi^{4} \alpha_{4}$ & $\xi^{2} \alpha_{1}$ & $\xi^{4} \alpha_{2}$ \\
$b$ & $e_{2}$ & $e_{3}$ & $e_{4}$ & $e_{1}$ & $e_{2^{\prime}}$ & $e_{3^{\prime}}$ & $e_{4^{\prime}}$ & $e_{1^{\prime}}$ & $\alpha_{2}$ & $\alpha_{3}$ & $\alpha_{4}$ & $\alpha_{1}$ \\
& & & $\alpha_{1}^{*}$ & $\alpha_{2}^{*}$ & $\alpha_{3}^{*}$ & $\alpha_{4}^{*}$ & $\sigma_{1}$ & $\sigma_{2}$ & $\sigma_{3}$ & $\sigma_{4}$ \\
\cline { 2 - 10 } & & & $\xi^{2} \alpha_{3}^{*}$ & $\xi^{4} \alpha_{4}^{*}$ & $\xi^{2} \alpha_{1}^{*}$ & $\xi^{4} \alpha_{2}^{*}$ & $\sigma_{3}$ & $\sigma_{4}$ & $\sigma_{1}$ & $\sigma_{2}$ & \\
& & & $\alpha_{2}^{*}$ & $\alpha_{3}^{*}$ & $\alpha_{4}^{*}$ & $\alpha_{1}^{*}$ & $\sigma_{2}$ & $\sigma_{3}$ & $\sigma_{4}$ & $\sigma_{1}$ &
\end{tabular}

where $\xi$ is a primitive 6 -th root of unity. Then, one can check that $\widehat{\widehat{Q}}=Q$.

3.3. Consider the admissible action of finite abelian group $G$ on $\mathbb{k} \widehat{Q}$ induced from the action of $G$ on $\mathbb{k} Q$ as the discussion above, we set

$$
\begin{array}{ll}
F^{\prime}:=(\mathbb{k} Q * G) * G \otimes_{\mathbb{k} Q * G}-: \quad \bmod -\mathbb{k}_{k} Q * G \longrightarrow \bmod -(\mathbb{k} Q * G) * G, \\
H^{\prime}:=\left.\operatorname{Res}\right|_{\mathbb{k} Q * G}: & \bmod -(\mathbb{k} Q * G) * G \longrightarrow \bmod -\mathbb{k}_{k} Q * G .
\end{array}
$$


Similar to the functors $F$ and $H$, one can check that $\left(H^{\prime}, F^{\prime}\right)$ and $\left(F^{\prime}, H^{\prime}\right)$ are adjoint pairs. Note that the Morita equivalence $\bmod -\mathbb{k}_{k} Q \rightarrow \bmod -\left(\mathbb{k}_{k} Q * G\right) * G$ is given by $\mathcal{M}:={ }_{(\mathrm{k} Q * G) * G} \mathbb{k} Q * G \otimes_{\mathbb{k} Q}-$, we have:

Lemma 3.8. There are natural isomorphisms

$$
F \cong H^{\prime} \mathcal{M} \quad \text { and } \quad F^{\prime} \cong \mathcal{M} H .
$$

Proof. First, $H^{\prime} \mathcal{M}={ }_{k} Q * G \mathbb{k} Q * G \otimes_{\mathbb{k} Q}-=F$ is clear. Next, since $\left(H^{\prime}, F^{\prime}\right)$ is an adjoint pair, for any $\mathbb{k}_{k} Q$-module $X$ and $\mathbb{k}_{\mathrm{k}} Q * G$-module $Y$, we have

$$
\begin{aligned}
\operatorname{Hom}_{\mathbb{k} Q}\left(X, \mathcal{M}^{-1} F^{\prime}(Y)\right) & \cong \operatorname{Hom}_{(\mathbb{k} Q * G) * G}\left(\mathcal{M}(X), F^{\prime}(Y)\right) \\
& \cong \operatorname{Hom}_{\mathbb{k} Q * G}\left(H^{\prime} \mathcal{M}(X), Y\right) \cong \operatorname{Hom}_{\mathbb{k} Q * G}(F(X), Y) .
\end{aligned}
$$

This implies that $\left(F, \mathcal{M}^{-1} F^{\prime}\right)$ is an adjoint pair and so that $H \cong \mathcal{M}^{-1} F^{\prime}$, $F^{\prime} \cong \mathcal{M} H$.

By Lemma 3.8 and [18, Proposition 1.8], we have the following proposition immediately.

Proposition 3.9. Let $X$ and $Y$ be indecomposable $\mathbb{k} Q * G$-modules. Then

(1) $F H(X) \cong H^{\prime} F^{\prime}(X) \cong \bigoplus_{g \in G}{ }^{g} X$;

(2) $H(X) \cong H(Y)$ if and only if $F^{\prime}(X) \cong F^{\prime}(Y)$, if and only if $Y \cong{ }^{g} X$ for some $g \in G$;

(3) $H(X)\left(\right.$ or $\left.F^{\prime}(X)\right)$ has exactly $\left|H_{X}\right|$ indecomposable summands.

Remark 3.10. Consider the action of $G$ on $\mathbb{k} Q * G$, we denote by

$$
H_{X}:=\left\{g \in G \mid F_{g}(X) \cong X\right\}
$$

and by $G_{X}$ a complete set of left coset representatives of $H_{X}$ in $G$, for any $X \in \bmod -\mathbb{k}_{k} Q * G$. In [10], we have shown that the number of indecomposable summands of $F^{\prime}(X)$ is just $\left|H_{X}\right|$ whenever $G$ is abelian (see [10, Theorem 1.2]). This means that $H(X)$ has $\left|H_{X}\right|$ indecomposable summands. Note that $H(X)$ is an indecomposable $G$-invariant $\mathbb{k}_{k} Q$-module, there exists a unique indecomposable $\mathbb{k}_{\mathrm{k}} Q$-module $M$ such that $H(X) \cong \sum(M)$. Therefore, we have $\left|H_{X}\right|=\left|G_{M}\right|$ and $\left|G_{X}\right|=\left|H_{M}\right|$. Following from Proposition 3.9(2), for an indecomposable $\mathbb{k}_{k} Q$-module $M$, there are $\left|G_{X}\right|=\left|H_{M}\right|$ non-isomorphic indecomposable $\mathbb{k}_{k} Q * G$-module structures on $\sum(M)$. This coincides with the result in $[10]$.

For the generalized McKay quiver $\widehat{Q}$, we denote by $(-,-)_{\widehat{Q}}$ the bilinear form on $\mathbb{Z} \widehat{I}$ determined by $\widehat{A}$, by $\Delta_{\widehat{Q}}$ the root system of $\widehat{Q}$ with simple roots $\varepsilon_{i \rho},(i, \rho) \in \widehat{I}$, and by $\mathcal{W}(\widehat{Q})$ the Weyl group of $\widehat{Q}$ with simple reflections $r_{i \rho}$, $(i, \rho) \in \widehat{I}$. Consider the map $h: \mathbb{Z} \widehat{I} \rightarrow \mathbb{Z I}$ defined above, we have:

Lemma 3.11. Let $\widehat{S}_{i}:=\prod_{\rho \in \operatorname{irr} G_{i}} r_{i \rho}$ for $i \in \mathcal{I}$. Then, for each $i \in \mathcal{I}$ and $\beta=\sum_{(i, \rho) \in \widehat{I}} \beta_{i \rho} \varepsilon_{i \rho} \in \mathbb{Z} \widehat{I}$, we have

(1) $\left(h(\beta), \bar{\varepsilon}_{i}\right)_{\Gamma}=d_{i} \sum_{\rho \in \operatorname{irr} G_{i}}\left(\beta, \varepsilon_{i \rho}\right)_{\widehat{Q}}$; 
(2) $h\left(\widehat{S}_{i}(\beta)\right)=\gamma_{i}(h(\beta))$;

(3) the map $\gamma_{i} \mapsto \widehat{S}_{i}$ induces an isomorphism $\mathcal{W}(\Gamma) \stackrel{\simeq}{\longrightarrow} C_{G}(\mathcal{W}(\widehat{Q}))$, the set of elements in $\mathcal{W}(\widehat{Q})$ commuting with the action of $G$.

Proof. (1) By the dual between $(Q, G)$ and $(\widehat{Q}, G)$, we obtain that

$$
b_{i j}=\sum_{\substack{i^{\prime} \in \mathcal{O}_{i} \\ j^{\prime} \in \mathcal{O}_{j}}} a_{i^{\prime} j^{\prime}}=\frac{d_{i} d_{j}}{|G|} \sum_{\substack{\rho \in \operatorname{irr} G i \\ \sigma \in \operatorname{irr} G_{j}}} a_{(i \rho)(j \sigma)},
$$

and so that

$$
b_{i j}=d_{i} \sum_{\rho \in \operatorname{irr} G_{i}} a_{(i \rho)(j \sigma)}
$$

for any $\sigma \in \operatorname{irr} G_{j}$. Therefore, we get

$$
\left(h(\beta), \bar{\epsilon}_{i}\right)_{\Gamma}=\sum_{i, j \in \mathcal{I}} b_{i j} h(\beta)_{j}=d_{i} \sum_{\substack{\rho \in \operatorname{irr} G_{i} \\ \sigma \in \operatorname{irr} G_{j}}} a_{(i \rho)(j \sigma)} \beta_{j \sigma}=d_{i} \sum_{\rho \in \operatorname{irr} G_{i}}\left(\beta, \varepsilon_{i \rho}\right)_{\widehat{Q}} .
$$

(2) Firstly, $\widehat{S}_{i}$ is well-defined since the action of $G$ on $\widehat{Q}$ is admissible. Secondly, it is easy to check that the bilinear form $(-,-) \widehat{Q}$ is $G$-invariant and $\widehat{S}_{i}$ commutes with the action of $G$. Thus, we have

$$
h\left(\widehat{S}_{i}(\beta)\right)=h(\beta)-\sum_{\rho \in \operatorname{irr} G_{i}}\left(\beta, \varepsilon_{i \rho}\right)_{\widehat{Q}} \bar{\varepsilon}_{i}=h(\beta)-\frac{1}{d_{i}}\left(h(\beta), \bar{\varepsilon}_{i}\right)_{\Gamma} \bar{\varepsilon}_{i}=\gamma_{i}(h(\beta)) .
$$

(3) By induction on the length, one can check that $C_{G}(\mathcal{W}(\widehat{Q}))$ is generated by $\widehat{S}_{i}, i \in \mathcal{I}$. Following from (2), we get $\gamma_{i} \mapsto S_{i}$ induces an isomorphism.

We are in a position to complete the proof of Theorem 1.1. We have shown that for any positive root $\alpha \in \Delta_{\Gamma}$, there exists an indecomposable $\widehat{Q}$ representation $\mathrm{X}$ such that $h(\operatorname{dim} X)=\alpha$. Moreover, if $\alpha$ is real, the number of $X$ (up to isomorphism) can be determined. Applying the technique in [12, Proposition 15], we have:

Proposition 3.12. The map $h: \Delta_{\widehat{Q}} \rightarrow \Delta_{\Gamma}$ is a surjection. If $\alpha \in \Delta_{\Gamma}$ is a positive real root, then there is a unique $G$-orbit of roots mapping to $\alpha$, and all of which are real.

Proof. Firstly, by Corollary 3.4, the map $h: \Delta_{\widehat{Q}} \rightarrow \Delta_{\Gamma}$ is well-defined. To show the surjectivity, we need to fine the preimages of all the fundamental roots in $\Delta_{\widehat{Q}}$.

We suppose that $\Gamma$ is connected. Then, for any $\alpha \in F_{\Gamma}$, we consider the set

$$
\mathcal{R}:=\left\{\beta \in \Delta_{\widehat{Q}} \mid \beta \text { is positive and } h(\beta) \leq \alpha\right\} .
$$

Since $\mathcal{R}$ is finite and non-empty, we take an element $\beta$ with maximal height. Suppose that $h(\beta)_{i}<\alpha_{i}$ for all $i \in \mathcal{I}$, then for any $\rho \in \operatorname{irr} G_{i}, h\left(\beta+\varepsilon_{i \rho}\right)=$ $h(\beta)+\bar{\varepsilon}_{i} \leq \alpha$. By the maximality of $\beta, \beta+\varepsilon_{i \rho}$ is not a root and so that $\left(\beta, \varepsilon_{i \rho}\right)_{\widehat{Q}} \geq 0$. Thus $\left(h(\beta), \bar{\varepsilon}_{i}\right)_{\Gamma} \geq 0$ for all $i \in \mathcal{I}$. We conclude that $h(\beta)$ and $\alpha$ 
have the same support, for otherwise, we can find such a vertex $(i, \rho)$ adjacent to the support of $\beta$ such that $\left(\beta, \varepsilon_{i \rho}\right)_{\widehat{Q}}<0$.

We take $\alpha \in F_{\Gamma}$ such that the support of $\alpha$ is $\mathcal{I}$, and set

$$
\Phi:=\left\{i \in \mathcal{I} \mid h(\beta)_{i}=\alpha_{i}\right\} .
$$

If $\Phi$ is the empty set, then $\beta+\varepsilon_{i \rho}$ is not a root for any vertex $(i, \rho) \in \widehat{Q}$, and so that the connected component of $\widehat{Q}$ which $\beta$ lies in is Dynkin (see $[13$, Proposition 4.9]). Therefore, $\widehat{Q}$ must be a disjoint union of copies of this Dynkin quiver, all in a single $G$-orbit. Thus $\widehat{Q}$ and $Q$ are representation finite [18], $\Gamma$ is a connected Dynkin diagram. This contradicts to that $\alpha$ is a imaginary root.

It follows that $\Phi$ is non-empty. We denote by $\widetilde{\Phi}$ the full subgraph of $\Gamma$ determined by $\Phi$. Let $T$ be a non-empty connected component of $\Gamma-\widetilde{\Phi}$, and let $\widetilde{\beta}$ be the restriction of $h(\beta)$ to $T$. If $T \neq \emptyset$, then for all vertices $j \in T$, we have $\left(\widetilde{\beta}, \bar{\varepsilon}_{j}\right)_{T} \geq\left(h(\beta), \bar{\varepsilon}_{j}\right)_{\Gamma} \geq 0$, where $(-,-)_{T}$ is the restriction of $(-,-)_{\Gamma}$ on $T$. Moreover, note that there exists a vertex $j \in T$ adjacent to $\widetilde{\Phi}$, we have $\left(\widetilde{\beta}, \bar{\varepsilon}_{j}\right)_{T}>0$. Therefore, $T$ is a Dynkin diagram [13, Corollary 4.9]. On the other hand, let $\widetilde{\beta}^{\prime}$ be the restriction of $\alpha-h(\beta)$ to $T$. Then $\widetilde{\beta}^{\prime}$ has the support $T$, and for any vertex $j \in T$,

$$
\left(\widetilde{\beta}^{\prime}, \bar{\varepsilon}_{j}\right)_{T}=\left(\alpha-h(\beta), \bar{\varepsilon}_{j}\right)_{\Gamma}=\left(\alpha, \bar{\varepsilon}_{j}\right)_{\Gamma}-\left(h(\beta), \bar{\varepsilon}_{j}\right)_{\Gamma} \leq 0 .
$$

Hence $T$ is not Dynkin. This is a contradiction. Therefore, $T$ is empty, $\widetilde{\Phi}=\Gamma$ and so that $h(\beta)=\alpha$. Thus, we have shown that $h$ is surjective by Lemma $3.11(3)$.

In general, assume that $\Gamma$ is non-connected. In this case, $F_{\Gamma}=\bigcup F_{\Gamma^{\prime}}$, where $\Gamma^{\prime}$ run over all connected components of $\Gamma$. By the discussion above, we see that any element $\alpha \in F_{\Gamma}$, there exists an element $\beta \in \Delta_{\widehat{Q}}$ such that $h(\beta)=\alpha$. Hence, $h$ is also surjective.

Finally, for any real root $\alpha \in \Delta_{\Gamma}$, we let $\beta \in \Delta_{\widehat{Q}}$ be the element such that $h(\beta)=\alpha$. Then, there is an element $\omega^{\prime} \in \mathcal{W}(\Gamma)$ and $i \in \mathcal{I}$ such that $\omega^{\prime}(\alpha)=\bar{\varepsilon}_{i}$. Let $\omega$ be the element in $C_{G}(\mathcal{W}(\widehat{Q}))$ corresponding to $\omega^{\prime}$. It follows that $\omega(\beta)$ must also be a simple root $\varepsilon_{i \rho}$ for some $\rho \in \operatorname{irr} G_{i}$. Therefore $\beta$ is real and uniquely determined up to a $G$-orbit.

Consider the action of $G$ on $\mathbb{k} \widehat{Q}$, any $g \in G$ also induces an additive autoequivalence functor $F_{g}: \bmod -\mathbb{k} \widehat{Q} \rightarrow \bmod -\mathbb{k} \widehat{Q}, X \mapsto{ }^{g} X$. Here we also denote by $G_{X}$ a complete set of left coset representatives of $H_{X}:=\{g \in G \mid$ $\left.F_{g}(X) \cong X\right\}$ in $G$, for any $X \in \bmod -k_{k} \widehat{Q}$. Following from Kac's Theorem, for any positive real root $\beta \in \Delta_{\widehat{Q}}$, there exists a unique $\widehat{Q}$-representation $X$ such that $\operatorname{dim} X=\beta$ and $H_{X}=H_{\beta}$. By Proposition 3.12, there are $\left|G_{X}\right|$ indecomposable $\widehat{Q}$-representations (up to isomorphism) such that the images of their dimension vector under the map $h$ are $\alpha$, if $h(\operatorname{dim} X)=\alpha$. Thus the proof of Theorem 1.1 is completed. 


\section{Proof of Theorem 1.2}

From now on, assume that $\mathbb{k}$ is an algebraically closed field with char $\mathbb{k}=0$, and $G \subseteq \operatorname{Aut}(\mathbb{k} Q)$ is a finite abelian group. In this section, we lift $G$ to $\bar{G} \subseteq \operatorname{Aut}(\mathfrak{g})$ such that the Kac-Moody algebra $\mathfrak{g}(\Gamma)$ can be embedded into the fixed point algebra $\mathfrak{g}^{\bar{G}}$. In this case, $\mathfrak{g}^{\bar{G}}$ is integrable as a $\mathfrak{g}(\Gamma)$-module.

Firstly, we recall some notations of Kac-Moody algebras. For a symmetricable generalized Cartan matrix $C=\left(c_{i j}\right)$ of size $n$ and rank $l$, there exist a diagonal matrix $D=\operatorname{diag}\left(d_{1}, \ldots, d_{n}\right)$ and a symmetric matrix $B=\left(b_{i j}\right)$ such that $C=D^{-1} B$. In fact, $d_{i}(1 \leq i \leq n)$ may be chosen to be positive integers. Let $\mathfrak{h}$ be a $2 n-l$ dimension $\mathbb{k}$-vector space. Choose linearly independent sets $\left\{H_{i} \in \mathfrak{h} \mid 1 \leq i \leq n\right\}$ and $\left\{\varepsilon_{i} \in \mathfrak{h}^{*} \mid 1 \leq i \leq n\right\}$ such that $\varepsilon_{j}\left(H_{i}\right)=c_{i j}$. Then the triple $\left(\mathfrak{h},\left\{\varepsilon_{i}\right\},\left\{H_{i}\right\}\right)_{1<i<n}$ is called a (minimal) realization of $C$. Since any two realizations of $C$ are isomorphic, there is a unique (up to isomorphism) Kac-Moody algebra $\mathfrak{g}(C)$ generated by $\mathfrak{h}, E_{i}, F_{i}, 1 \leq i \leq n$, with relations

$$
\begin{aligned}
& {\left[H, H^{\prime}\right]=0, \quad\left[H, E_{j}\right]=\varepsilon_{j}(H) E_{j}, \quad\left(\operatorname{ad} E_{i}\right)^{1-c_{i j}} E_{j}=0,} \\
& {\left[E_{i}, F_{j}\right]=\delta_{i j} H_{i}, \quad\left[H, F_{j}\right]=-\varepsilon_{j}(H) F_{j}, \quad\left(\operatorname{ad} F_{i}\right)^{1-c_{i j}} F_{j}=0 \text {, }}
\end{aligned}
$$

for any $H, H^{\prime} \in \mathfrak{h}$, where $\delta_{i j}$ is the Kronecker sign. Moreover, the center $\mathfrak{c}$ of $\mathfrak{g}(C)$ is given by

$$
\left\{H \in \mathfrak{h} \mid \varepsilon_{i}(H)=0 \text { for all } 1 \leq i \leq n\right\} \subseteq[\mathfrak{g}(C), \mathfrak{g}(C)] .
$$

For the details one can see [13].

For the pair $(Q, G)$, we have obtained the valued graph $\Gamma$ with symmetricable generalized Cartan matrix $C=\left(c_{i j}\right)$ of size $|\mathcal{I}|$ and the generalized McKay quiver $\widehat{Q}$ with symmetric generalized Cartan matrix $\widehat{A}=\left(a_{(i \rho)(j \sigma)}\right)$ of size $|\widehat{I}|$, see Section 2. Therefore we have Kac-Moody algebras $\mathfrak{g}(\Gamma):=\mathfrak{g}(C)$ corresponding to the realization $\left(\mathfrak{h}(\Gamma),\left\{\bar{\varepsilon}_{i}\right\},\left\{\bar{H}_{i}\right\}\right)$ of $C$ and $\mathfrak{g}:=\mathfrak{g}(\widehat{Q})=\mathfrak{g}(\widehat{A})$ corresponding to the realization $\left(\mathfrak{h},\left\{\varepsilon_{i \rho}\right\},\left\{H_{i \rho}\right\}\right)$ of $\widehat{A}$. Denote by $r$ and $s$ the coranks of $C$ and $\widehat{A}$, then $\operatorname{dim}_{\mathbb{k}} \mathfrak{h}(\Gamma)=|\mathcal{I}|+r$ and $\operatorname{dim}_{\mathbb{k}} \mathfrak{h}=|\widehat{I}|+s$.

We suppose that $\mathfrak{g}(\Gamma)$ is generated by $\mathfrak{h}(\Gamma)$ and $\bar{E}_{i}, \bar{F}_{i}, i \in \mathcal{I}$. There is a symmetric bilinear form $(-,-)_{\Gamma}$ on $\mathfrak{h}(\Gamma)$ such that

$$
\left(\bar{H}_{i}, \bar{H}\right)_{\Gamma}=\frac{1}{d_{i}} \bar{\varepsilon}_{i}(\bar{H})
$$

for all $\bar{H} \in \mathfrak{h}(\Gamma)$. Then we can extend it uniquely to an invariant non-degenerate symmetric bilinear form on $\mathfrak{g}(\Gamma)$ such that

$$
\left(\bar{E}_{i}, \bar{F}_{i}\right)_{\Gamma}=\frac{1}{d_{i}}
$$

Moreover, $(-,-)_{\Gamma}$ determines a bijection $\nu: \mathfrak{h}(\Gamma) \rightarrow \mathfrak{h}^{*}(\Gamma)$ sending $\bar{H}_{i}$ to $\frac{1}{d_{i}} \bar{\varepsilon}_{i}$, and hence induces a bilinear form on $\mathfrak{h}^{*}(\Gamma)$. We also denote this bilinear form by $(-,-)_{\Gamma}$. Note that $\left(\bar{\varepsilon}_{i}, \bar{\varepsilon}_{i}\right)_{\Gamma}=b_{i j}$. It recovers the bilinear form defined in 
Section 2.3 for the root lattice $\mathbb{Z} \mathcal{I}$. Similarly, there is a symmetric bilinear form on $\mathfrak{h}^{*}=\mathfrak{h}^{*}(\widehat{Q})$ with $\left(\varepsilon_{i \rho}, \varepsilon_{j \sigma}\right)_{\widehat{Q}}=a_{(i \rho)(j \sigma)}$.

We now consider the action of $G$ on the quiver $\widehat{Q}$ defined in Section 3.2. Recall that the derived algebra $\mathfrak{g}^{\prime}$ of $\mathfrak{g}$ is generated by $H_{i \rho}, E_{i \rho}, F_{i \rho},(i, \rho) \in \widehat{I}$ and the action of $G$ on $\widehat{Q}$ satisfies

$$
a_{(i \rho)(j \sigma)}=a_{\left(i \rho^{\prime}\right)\left(j \sigma^{\prime}\right)} \quad \text { if }\left(i, \rho^{\prime}\right)=g(i, \rho) \text { and }\left(j, \sigma^{\prime}\right)=g(j, \sigma)
$$

for some $g \in G$. Then, there is a natural action of $G$ on $\mathfrak{g}^{\prime}$ given by

$$
g\left(H_{i \rho}\right)=H_{i \rho^{\prime}}, \quad g\left(E_{i \rho}\right)=E_{i \rho^{\prime}}, \quad g\left(F_{i \rho}\right)=F_{i \rho^{\prime}}
$$

for any $g \in G$. Denote by $\mathfrak{h}^{\prime}(\Gamma)$ and $\mathfrak{h}^{\prime}$ the Cartan subalgebra of $\mathfrak{g}^{\prime}(\Gamma):=$ $[\mathfrak{g}(\Gamma), \mathfrak{g}(\Gamma)]$ and $\mathfrak{g}^{\prime}$ respectively. It is easy to see that the map

$$
\phi: \quad \mathfrak{h}^{\prime}(\Gamma) \rightarrow\left(\mathfrak{h}^{\prime}\right)^{G}
$$

given by $\phi\left(\bar{H}_{i}\right)=\sum_{\rho \in \operatorname{irr} G_{i}} H_{i \rho}$ is an isomorphism and

$$
\left(\bar{H}, \bar{H}^{\prime}\right)_{\Gamma}=\frac{1}{|G|}\left(\phi(\bar{H}), \phi\left(\bar{H}^{\prime}\right)\right)_{\widehat{Q}}
$$

for $\bar{H}, \bar{H} \in \mathfrak{h}^{\prime}(\Gamma)$. In particular, the fixed point subalgebra $\mathfrak{c}^{G}$ of the center of $\mathfrak{g}(\widehat{Q})$ is isomorphic to the center $\mathfrak{c}(\Gamma)$ of $\mathfrak{g}(\Gamma)$.

We wish to extend the action of $G$ on $\mathfrak{g}^{\prime}$ to the whole Lie algebra $\mathfrak{g}$. Let $\operatorname{Aut}(\widehat{A})$ denote the set of permutations $g$ of $\widehat{I}$ satisfying

$$
a_{(i \rho)(j \sigma)}=a_{\left(l \rho^{\prime}\right)\left(k \sigma^{\prime}\right)} \quad \text { if }\left(l, \rho^{\prime}\right)=g(i, \rho) \text { and }\left(k, \sigma^{\prime}\right)=g(j, \sigma) .
$$

Let DAut $(\mathfrak{g})$ denote the subgroup of $\operatorname{Aut}(\mathfrak{g})$ consisting of the automorphisms preserving each of the sets $\mathfrak{h},\left\{E_{i \rho}\right\}$ and $\left\{F_{i \rho}\right\}$.

Proposition 4.1 (see [14, Section 4.19]). There is a short exact sequence

$$
0 \rightarrow \operatorname{Hom}_{\mathbb{k}}\left(\mathfrak{h} / \mathfrak{h}^{\prime}, \mathfrak{c}\right) \longrightarrow \operatorname{DAut}(\mathfrak{g}) \longrightarrow \operatorname{Aut}(\widehat{A}) \rightarrow 0 .
$$

Proof. It is easy to see that $\bar{g}\left(H_{i \rho}\right)=H_{j \sigma}, \bar{g}\left(E_{i \rho}\right)=E_{j \sigma}$ and $\bar{g}\left(F_{i \rho}\right)=F_{j \sigma}$ for any $\bar{g} \in \operatorname{DAut}(\mathfrak{g})$. Thus, there exists a unique permutation $g \in \operatorname{Aut}(\widehat{A})$ corresponding to $\bar{g}$ such that $(j, \sigma)=g(i, \rho)$. Moreover, each $g \in \operatorname{Aut}(\widehat{A})$ can be obtained in this way.

Let $\Lambda:=\mathbb{k} \widehat{I}$ be the subspace of $\mathfrak{h}^{*}$ spanned by $\left\{\varepsilon_{i \rho} \mid(i, \rho) \in \widehat{I}\right\}$. Then there is a natural action of $\operatorname{Aut}(\widehat{A})$ on $\Lambda: g\left(\varepsilon_{i \rho}\right)=\varepsilon_{j \sigma}$, where $(j, \sigma)=g(i, \rho), g \in G$, and it induces an action of $\operatorname{Aut}(\widehat{A})$ on the quotient space $\mathfrak{h} / \mathfrak{c}$ since $\mathfrak{h} / \mathfrak{c}$ is dual to $\Lambda$. It maps $H_{i \rho} \bmod \mathfrak{c}$ to $H_{j \sigma} \bmod \mathfrak{c}$, and so that $\mathfrak{h}^{\prime} / \mathfrak{c}$ is $\operatorname{Aut}(\widehat{A})$-stable. Since $\operatorname{Aut}(\widehat{A})$ is finite, there exists $\mathfrak{h}^{\prime \prime}$ such that $\mathfrak{h}=\mathfrak{h}^{\prime} \oplus \mathfrak{h}^{\prime \prime}$ and $\left(\mathfrak{h}^{\prime \prime}+\mathfrak{c}\right) / \mathfrak{c}$ is $\operatorname{Aut}(\widehat{A})$ stable. For any $g \in \operatorname{Aut}(\widehat{A})$, we can define an automorphism $\bar{g} \in \operatorname{DAut}(\mathfrak{g})$ by

$$
\bar{g}\left(H_{i \rho}\right)=H_{j \sigma}, \quad \bar{g}\left(E_{i \rho}\right)=E_{j \sigma} \quad \text { and } \quad \bar{g}\left(F_{i \rho}\right)=F_{j \sigma},
$$

and $\left.\bar{g}\right|_{\mathfrak{h}^{\prime \prime}}$ is the pull-back of $g$ on $\left(\mathfrak{h}^{\prime \prime}+\mathfrak{c}\right) / \mathfrak{c}$. 
Clearly, the kernel of the map DAut $(\mathfrak{g}) \rightarrow \operatorname{Aut}(\widehat{A})$ is the subgroup $\operatorname{Aut}\left(\mathfrak{g} ; \mathfrak{g}^{\prime}\right)$ consisting of all automorphisms acting trivially on $\mathfrak{g}^{\prime}$. One can check that an automorphism $\alpha \in \operatorname{Aut}\left(\mathfrak{g} ; \mathfrak{g}^{\prime}\right)$ if and only if there exists a map $\varphi: \mathfrak{h}^{\prime \prime} \rightarrow \mathfrak{c}$ such that $\alpha(H)=H+\varphi(H)$ for all $H \in \mathfrak{h}^{\prime \prime}$. Thus, there are isomorphisms $\operatorname{Aut}\left(\mathfrak{g} ; \mathfrak{g}^{\prime}\right) \cong \operatorname{Hom}_{\mathbb{k}}\left(\mathfrak{h}^{\prime \prime}, \mathfrak{c}\right) \cong \operatorname{Hom}_{\mathbb{k}}\left(\mathfrak{h} / \mathfrak{h}^{\prime}, \mathfrak{c}\right)$.

Therefore, for each $\alpha \in \operatorname{Aut}\left(\mathfrak{g} ; \mathfrak{g}^{\prime}\right)$ and $g \in \operatorname{Aut}(\widehat{A})$, we have an element $\bar{g} \in \operatorname{DAut}(\mathfrak{g})$ by setting $\left.\bar{g}\right|_{\mathfrak{g}^{\prime}}=g$ and $\left.\bar{g}\right|_{\mathfrak{h}^{\prime \prime}}=\alpha$. Moreover, for any $\alpha \in \operatorname{Aut}\left(\mathfrak{g} ; \mathfrak{g}^{\prime}\right)$ corresponding to $\varphi: \mathfrak{h}^{\prime \prime} \rightarrow \mathfrak{c}$, it is easy to see that $\alpha^{t}(H)=H+t \varphi(H)$ for any $t \in \mathbb{Z}$ and $H \in \mathfrak{h}^{\prime \prime}$. That is to say, an automorphism $\alpha \in \operatorname{Aut}\left(\mathfrak{g} ; \mathfrak{g}^{\prime}\right)$ has finite order if and only if the corresponding map $\varphi: \mathfrak{h}^{\prime \prime} \rightarrow \mathfrak{c}$ is zero.

We now fix $\Omega=\left\{g_{1}, g_{2}, \ldots, g_{n}\right\}$ a set of generators of $G$. We can view $G$ as a finite abelian subgroup of $\operatorname{Aut}(\widehat{A})$. By Proposition 4.1, we can lift $G$ to an automorphism group $\bar{G}=\{\bar{g} \mid g \in G\}$ of $\mathfrak{g}$ corresponding to a set of linear maps $\left\{\varphi_{i}=\varphi_{g_{i}}: \mathfrak{h}^{\prime \prime} \rightarrow \mathfrak{c} \mid g_{i} \in \Omega\right\}$. It is easy to see that for any $H \in \mathfrak{h}$, we have $\varepsilon_{i \rho^{\prime}}(\bar{g}(H))=\varepsilon_{i \rho}(H)$ if $\left(i, \rho^{\prime}\right)=g(i, \rho)$. Let

$$
\mathcal{S}:=\operatorname{span}\left\{\varepsilon_{i \rho}-\varepsilon_{i \rho^{\prime}} \mid i \in \mathcal{I}, \rho, \rho^{\prime} \in \operatorname{irr} G_{i}\right\} \subseteq \mathfrak{h}^{*}
$$

and

$$
\mathcal{H}:=\left\{H \in \mathfrak{h} \mid \varepsilon_{i \rho}(H)=\varepsilon_{i \rho^{\prime}}(H) \text { for all } \rho, \rho^{\prime} \in \operatorname{irr} G_{i} \text { and } i \in \mathcal{I}\right\}=\operatorname{ann}_{\mathfrak{h}} \mathcal{S} .
$$

Then $\mathcal{H}$ contains the center $\mathfrak{c}, \mathcal{H} / \mathfrak{c}=(\mathfrak{h} / \mathfrak{c})^{G}$ and so that, for any lifting $\bar{G}$ of $G, \mathcal{H}^{\bar{G}}=\mathfrak{h}^{\bar{G}}$.

Lemma 4.2. $\mathcal{H}$ has $\mathbb{k}$-dimension $|\mathcal{I}|+s, \mathcal{H} \cap \mathfrak{h}^{\prime}$ has $\mathbb{k}$-dimension $|\mathcal{I}|+s-r$ and therefore $\mathcal{H} \cap \mathfrak{h}^{\prime \prime}$ has $\mathbb{k}$-dimension $r$.

Proof. Fix a $\rho \in \operatorname{irr} G_{i}$, note that

$$
\left\{\varepsilon_{i \rho}-\varepsilon_{i \rho^{\prime}} \mid i \in \mathcal{I}, \rho^{\prime} \in \operatorname{irr} G_{i} \backslash \rho\right\}
$$

is a basis of $\mathcal{S}$, we obtain that $\operatorname{dim}_{\mathbb{k}} \mathcal{H}=\operatorname{dim}_{\mathbb{k}} \mathfrak{h}-\operatorname{dim}_{\mathbb{k}} \mathcal{S}=|\mathcal{I}|+s$. Since $\left(\mathcal{H} \cap \mathfrak{h}^{\prime}\right) / \mathfrak{c}=\left(\mathfrak{h}^{\prime} / \mathfrak{c}\right)^{G}$ is isomorphic to $\left(\mathfrak{h}^{\prime}\right)^{G} / \mathfrak{c}^{G}, \operatorname{dim}_{\mathbb{k}}\left(\mathfrak{h}^{\prime}\right)^{G}=|\mathcal{I}|$ and $\operatorname{dim}_{\mathbb{k}} \mathfrak{c}^{G}=$ $\operatorname{dim}_{\mathbb{k}} \mathfrak{c}(\Gamma)=r, \mathcal{H} \cap \mathfrak{h}^{\prime}$ has $\mathbb{k}$-dimension $|\mathcal{I}|+s-r$ and so that $\mathcal{H} \cap \mathfrak{h}^{\prime \prime}$ has kk-dimension $r$.

Proposition 4.3. Let $\bar{G}$ be a lifting of $G$ to $\mathfrak{g}$ corresponding to $\left\{\varphi_{i}: \mathfrak{h}^{\prime \prime} \rightarrow \mathfrak{c} \mid\right.$ $1 \leq i \leq n\}$. Then

$$
\left(\mathcal{H}^{\bar{G}},\left\{\frac{d_{i}}{|G|} \sum_{\rho \in \operatorname{irr} G_{i}} \varepsilon_{i \rho}\right\},\left\{\sum_{\rho \in \operatorname{irr} G_{i}} H_{i \rho}\right\}\right)
$$

is a realization of $C$ if and only if $\varphi_{i}\left(\mathcal{H} \cap \mathfrak{h}^{\prime \prime}\right)=0$ for all $1 \leq i \leq n$.

Proof. We denote by

$$
H_{i}:=\sum_{\rho \in \operatorname{irr} G_{i}} H_{i \rho} \quad \text { and } \quad \epsilon_{i}:=\frac{d_{i}}{|G|} \sum_{\rho \in \operatorname{irr} G_{i}} \varepsilon_{i \rho}
$$


for all $i \in \mathcal{I}$. Since $\left\{H_{i} \mid i \in \mathcal{I}\right\}$ is a basis of $\left(\mathcal{H} \cap \mathfrak{h}^{\prime}\right)^{G}, \mathcal{H}^{\bar{G}}$ has dimension $|\mathcal{I}|+r$ if and only if there are $h_{1}^{\prime}, h_{2}^{\prime}, \ldots, h_{r}^{\prime} \in \mathcal{H}^{\bar{G}}$ spanning the complementary space of $\left(\mathcal{H} \cap \mathfrak{h}^{\prime}\right)^{G}$ in $\mathcal{H}^{\bar{G}}$.

Since $\left(\mathfrak{h}^{\prime \prime}+\mathfrak{c}\right) / \mathfrak{c}$ is $G$-stable, $\left(\left(\mathfrak{h}^{\prime \prime}+\mathfrak{c}\right) / \mathfrak{c}\right)^{G}$ has $\mathbb{k}$-dimension $r$ by Lemma 4.2. We can find linearly independent elements $h_{1}^{\prime \prime}, h_{2}^{\prime \prime}, \ldots, h_{r}^{\prime \prime} \in \mathcal{H} \cap \mathfrak{h}^{\prime \prime}$ such that $h_{i}^{\prime \prime}$ $\bmod \mathfrak{c}$ are fixed by $G$. Since $\varphi_{i}\left(\mathcal{H} \cap \mathfrak{h}^{\prime \prime}\right)=0$ for all $i, h_{1}^{\prime \prime}, h_{2}^{\prime \prime}, \ldots, h_{r}^{\prime \prime}$ are $G$-stable and form a basis of $\mathcal{H} \cap \mathfrak{h}^{\prime \prime}$. Therefore, we take $h_{i}^{\prime}=h_{i}^{\prime \prime}$ for all $1 \leq i \leq r$. On the other hand, if we can find such elements $h_{1}^{\prime}, h_{2}^{\prime}, \ldots, h_{r}^{\prime}$, then each $h_{i}^{\prime \prime}$ has the form

$$
h_{i}^{\prime \prime}=\sum_{j=1}^{s} p_{i j} h_{j}^{\prime}-\sum_{(j, \sigma) \in \widehat{I}} q_{i(j \sigma)} H_{j \sigma}
$$

for some $p_{i j}, q_{i(j \sigma)} \in \mathbb{k}$, and

$$
\begin{aligned}
\varphi_{l}\left(h_{i}^{\prime \prime}\right) & =\bar{g}_{l}\left(\sum_{j=1}^{s} p_{i j} h_{j}^{\prime}-\sum_{(j, \sigma) \in \widehat{I}} q_{i(j \sigma)} H_{j \sigma}\right)-\sum_{j=1}^{s} p_{i j} h_{j}^{\prime}+\sum_{(j, \sigma) \in \widehat{I}} q_{i(j \sigma)} H_{j \sigma} \\
& =\sum_{(j, \sigma) \in \widehat{I}} q_{i(j \sigma)}\left(H_{j \sigma}-H_{j \sigma^{1}}\right),
\end{aligned}
$$

where $\left(j, \sigma^{1}\right)=g_{l}(j, \sigma)$. It follows that

$$
t \varphi_{l}\left(h_{i}^{\prime \prime}\right)=\sum_{(j, \sigma) \in \widehat{I}} q_{i(j \sigma)}\left(H_{j \sigma}-H_{j \sigma^{t}}\right)
$$

for any $t \in \mathbb{Z}$, where $\left(j, \sigma^{t}\right)=g_{l}^{t}(j, \sigma)$. Note that $\widehat{I}$ is a finite set, there exist some $t \in \mathbb{Z}$ such that $g_{l}^{t}(j, \sigma)=(j, \sigma)$ for all $(j, \sigma) \in \widehat{I}$, and so that $t \varphi_{l}\left(h_{i}^{\prime \prime}\right)=0$, $\varphi_{l}\left(h_{i}^{\prime \prime}\right)=0$ for all $i$ and $l$. Thus $\varphi_{i}\left(\mathcal{H} \cap \mathfrak{h}^{\prime \prime}\right)=0$ for any $1 \leq i \leq n$.

Since

$$
\epsilon_{j}\left(H_{i}\right)=\frac{d_{i}}{|G|} \sum_{\substack{\rho \in \operatorname{irr} G_{i} \\ \sigma \in \operatorname{irr} G_{j}}} \varepsilon_{j \sigma}\left(H_{i \rho}\right)=\frac{d_{i}}{|G|} \sum_{\substack{\rho \in \operatorname{irr} G_{i} \\ \sigma \in \operatorname{irr} G_{j}}} a_{(i \rho)(j \sigma)}=c_{i j}
$$

and $H_{i}(i \in \mathcal{I})$ are linearly independent, it remains to show $\epsilon_{i}, i \in \mathcal{I}$ are linearly independent modulo $\operatorname{ann}_{\mathfrak{h}^{*}}\left(\mathcal{H}^{\bar{G}}\right)$. Let

$$
\epsilon:=\sum_{j \in \mathcal{I}} \mu_{j} \epsilon_{j} \in \operatorname{ann}_{\mathfrak{h}^{*}}\left(\mathcal{H}^{\bar{G}}\right), \quad \mu_{j} \in \mathbb{k} .
$$

Then

$$
0=\epsilon\left(H_{i}\right)=\sum_{j \in \mathcal{I}} \mu_{j} \epsilon_{j}\left(H_{i}\right)=\sum_{j \in \mathcal{I}} c_{i j} \mu_{j}
$$

for all $i \in \mathcal{I}$, and so that

$$
\epsilon\left(H_{i \rho}\right)=\sum_{j \in \mathcal{I}} \mu_{j} \epsilon_{j}\left(H_{i \rho}\right)=\frac{1}{|G|} \sum_{j \in \mathcal{I}} b_{i j} \mu_{j}=\frac{d_{i}}{|G|} \sum_{j \in \mathcal{I}} c_{i j} \mu_{j}=0
$$


for all $(i, \rho) \in \widehat{I}$. Therefore,

$$
\epsilon \in \operatorname{ann}_{\mathfrak{h} *}\left(\mathcal{H}^{\bar{G}}+\mathfrak{h}^{\prime}\right)=\operatorname{ann}_{\mathfrak{h} *}\left(\mathcal{H}+\mathfrak{h}^{\prime}\right) \subseteq \operatorname{ann}_{\mathfrak{h} *}(\mathcal{H})=\mathcal{S} .
$$

It is equivalent to say

$$
\mu_{j} \epsilon_{j}=\frac{d_{j} \mu_{j}}{|G|} \sum_{\sigma \in \operatorname{irr} G_{j}} \varepsilon_{j \sigma} \in \operatorname{span}\left\{\varepsilon_{j \rho}-\varepsilon_{j \rho^{\prime}} \mid \rho, \rho^{\prime} \in \operatorname{irr} G_{j}\right\}
$$

for each $j \in \mathcal{I}$. It concludes that $\mu_{j}=0$ for all $j \in \mathcal{I}$, and so that $\epsilon_{j}$ are linearly independent in $\left(\mathcal{H}^{\bar{G}}\right)^{*}$. The proof is completed.

Remark 4.4. Since

$$
\operatorname{Hom}_{\mathbb{k}}\left(\mathfrak{h}^{\prime \prime}, \mathfrak{c}\right) \cong \operatorname{Hom}_{\mathbb{k}}\left(\mathfrak{h} / \mathfrak{h}^{\prime}, \mathfrak{c}\right),
$$

for any lifting $\bar{G}$ of $G$, there exists a family of maps $\left\{\psi_{i}=\psi_{g_{i}}: \mathfrak{h} / \mathfrak{h}^{\prime} \rightarrow \mathfrak{c}\right.$ | $\left.g_{i} \in \Omega\right\}$ corresponding to it. Moreover, it is easy to see that the condition $\varphi_{i}\left(\mathcal{H} \cap \mathfrak{h}^{\prime \prime}\right)=0$ is equivalent to $\psi_{i}\left(\left(\mathcal{H}+\mathfrak{h}^{\prime}\right) / \mathfrak{h}^{\prime}\right)=0$.

Now we can prove the main results of this section.

Proposition 4.5. There is a monomorphism $\mathfrak{g}^{\prime}(\Gamma) \rightarrow\left(\mathfrak{g}^{\prime}\right)^{G}$, and for the lifting $\bar{G}$ of $G$ corresponding to $\left\{\varphi_{i}: \mathfrak{h}^{\prime \prime} \rightarrow \mathfrak{c} \mid 1 \leq i \leq n\right\}$ with $\varphi_{i}\left(\mathcal{H} \cap \mathfrak{h}^{\prime \prime}\right)=0$, we can extend this monomorphism to the whole Lie algebra such that

$$
\mathfrak{g}(\Gamma) \rightarrow \mathfrak{g}^{\bar{G}}
$$

is also a monomorphism.

Proof. We set

$$
H_{i}:=\sum_{\rho \in \operatorname{irr} G_{i}} H_{i \rho}, \quad E_{i}:=\sum_{\rho \in \operatorname{irr} G_{i}} E_{i \rho}, \quad F_{i}:=\sum_{\rho \in \operatorname{irr} G_{i}} F_{i \rho}
$$

for all $i \in \mathcal{I}$. Then $H_{i}, E_{i}, F_{i} \in\left(\mathfrak{g}^{\prime}\right)^{G}$ and

$$
\begin{aligned}
& {\left[H_{i}, H_{j}\right]=0,} \\
& {\left[E_{i}, F_{j}\right]=\sum_{\substack{\rho \in \operatorname{irr} G_{i} \\
\sigma \in \operatorname{irr} G_{j}}}\left[E_{i \rho}, F_{j \sigma}\right]=\delta_{i j} \sum_{\rho \in \operatorname{irr} G_{i}} H_{i \rho}=\delta_{i j} H_{i},} \\
& {\left[H_{i}, E_{j}\right]=\sum_{\substack{\rho \in \operatorname{irr} G_{i} \\
\sigma \in \operatorname{irr} G_{j}}}\left[H_{i \rho}, E_{j \sigma}\right]=\sum_{\substack{\rho \in \operatorname{irr} G_{i} \\
\sigma \in \operatorname{irr} G_{j}}} a_{(i \rho)(j \sigma)} E_{j \sigma}=c_{i j} \sum_{\sigma \in \operatorname{irr} G_{j}} E_{j \sigma}=c_{i j} E_{j} .}
\end{aligned}
$$

Similarly, we have $\left[H_{i}, F_{j}\right]=c_{i j} F_{j}$ for any $i, j \in \mathcal{I}$. Note that $\operatorname{ad} E_{i \rho}$ and $\operatorname{ad} E_{i \rho^{\prime}}$ commute for any $\rho, \rho^{\prime} \in \operatorname{irr} G_{i}$, we have

$$
\left(\operatorname{ad} E_{i}\right)^{n}=\sum_{\lambda} \Phi_{\lambda}^{n} \prod_{\rho \in \operatorname{irr} G_{i}}\left(\operatorname{ad} E_{i \rho}\right)^{\lambda_{\rho}}
$$

for any positive integer $n$, where $\lambda$ takes though all the sequence $\lambda=\left(\lambda_{\rho}\right)_{\rho \in \operatorname{irr} G_{i}}$ satisfying

$$
\sum_{\rho \in \operatorname{irr} G_{i}} \lambda_{\rho}=n
$$


and the combinatorial number

$$
\Phi_{\lambda}^{n}=\left(\begin{array}{c}
n \\
\rho_{1}
\end{array}\right)\left(\begin{array}{c}
n-\rho_{1} \\
\rho_{2}
\end{array}\right) \cdots\left(\begin{array}{c}
n-\rho_{1}-\cdots-\rho_{\left|\operatorname{irr} G_{i}\right|-1} \\
\rho_{\left|\operatorname{irr} G_{i}\right|}
\end{array}\right)
$$

for any $\lambda=\left(\rho_{1}, \rho_{2}, \ldots, \rho_{\left|\operatorname{irr} G_{i}\right|}\right)$. In particular, if $n=1-c_{i j}$, then $\lambda_{\rho}>$ $1-a_{(i \rho)(j \sigma)}$ for some $\rho \in \operatorname{irr} G_{i}$ and so that

$$
\left(\operatorname{ad} E_{i \rho}\right)^{\lambda_{\rho}} E_{j \sigma}=0, \quad\left(\operatorname{ad} E_{i}\right)^{1-c_{i j}} E_{j}=0 .
$$

Similarly, $\left(\operatorname{ad} F_{i}\right)^{1-c_{i j}} F_{j}=0$ for any $i, j \in \mathcal{I}$. Therefore, there exists a non-zero homomorphism $\mathfrak{g}^{\prime}(\Gamma) \rightarrow\left(\mathfrak{g}^{\prime}\right)^{G}$.

Since $\left(\mathcal{H}^{\bar{G}},\left\{\frac{d_{i}}{|G|} \phi\left(\varepsilon_{i}\right)\right\},\left\{\phi\left(H_{i}\right)\right\}\right)$ is a realization of $C$ by Proposition 4.3, there is an isomorphism $\mathfrak{h}(\Gamma) \rightarrow \mathcal{H}^{\bar{G}}, \bar{H}_{i} \rightarrow H_{i}$. Therefore we can get a homomorphism $\mathfrak{g}(\Gamma) \rightarrow \mathfrak{g}^{\bar{G}}$ by compositing the homomorphisms $\mathfrak{g}^{\prime}(\Gamma) \rightarrow\left(\mathfrak{g}^{\prime}\right)^{G}$ and $\mathfrak{h}(\Gamma) \rightarrow \mathcal{H}^{\bar{G}}$. By [13, Proposition 1.7(b)], $\mathfrak{g}(\Gamma) \rightarrow \mathfrak{g}^{\bar{G}}$ and $\mathfrak{g}^{\prime}(\Gamma) \rightarrow\left(\mathfrak{g}^{\prime}\right)^{G}$ are monomorphisms.

Now, we can identify $\mathfrak{g}(\Gamma)$ with a subalgebra of $\mathfrak{g}^{\bar{G}}$. Following from Section 3.1 , the map

$$
h: \quad \mathbb{Z} \widehat{I} \rightarrow \mathbb{Z} \mathcal{I}, \quad \beta \mapsto h(\beta), \quad h(\beta)_{i}=\sum_{\rho \in \operatorname{irr} G_{i}} \beta_{i \rho},
$$

satisfies

$$
d_{i}\left(\beta, \sum_{\rho \in \operatorname{irr} G_{i}} \varepsilon_{i \rho}\right)_{\widehat{Q}}=\left(h(\beta), \bar{\varepsilon}_{i}\right)_{\Gamma}
$$

for all $\beta=\sum_{(i, \rho) \in \widehat{I}} \beta_{i \rho} \varepsilon_{i \rho} \in \mathbb{Z} \widehat{I}$ and $h\left(\Delta_{\widehat{Q}}\right)=\Delta_{\Gamma}$ by Proposition 3.12 .

Proposition 4.6. The monomorphism $\mathfrak{g}(\Gamma) \rightarrow \mathfrak{g}^{\bar{G}}$ endows $\mathfrak{g}^{\bar{G}}$ with an integrable $\mathfrak{g}(\Gamma)$-module structure under the adjoint action of $\mathfrak{g}(\Gamma)$.

Proof. Firstly, we identity the realization $\left(\mathfrak{h}(\Gamma),\left\{\bar{\varepsilon}_{i}\right\},\left\{\bar{H}_{i}\right\}\right)$ with $\left(\mathcal{H}^{\bar{G}},\left\{\epsilon_{i}\right\}\right.$, $\left.\left\{H_{i}\right\}\right)$. For any non-zero $\beta=\sum_{(i, \rho) \in \widehat{I}} \beta_{i \rho} \varepsilon_{i \rho} \in \Delta_{\widehat{Q}}$ and $H \in \mathcal{H}^{\bar{G}}$, we have

$$
\varepsilon_{i \rho}(H)=\frac{d_{i}}{|G|} \sum_{\rho \in \operatorname{irr} G_{i}} \varepsilon_{i \rho}(H)=\bar{\varepsilon}_{i}(H)
$$

and

$\beta(H)=\sum_{(i, \rho) \in \widehat{I}} \beta_{i \rho} \varepsilon_{i \rho}(H)=\sum_{i \in \mathcal{I}}\left(\sum_{\rho \in \operatorname{irr} G_{i}} \beta_{i \rho}\right) \bar{\varepsilon}_{i}(H)=\sum_{i \in \mathcal{I}} h(\beta)_{i} \bar{\varepsilon}_{i}(H)=h(\beta)(H)$.

Denote by $H_{\beta}=\{g \in G \mid g(\beta)=\beta\}$ and $G_{\beta}$ a complete set of left coset representatives of $H_{\beta}$ in $G$. Then $H_{\beta}$ acts on the root space $\mathfrak{g}_{\beta}$. Suppose that $x \in \mathfrak{g}_{\beta}$ satisfies $g(x)=x$ for any $g \in H_{\beta}$. Let

$$
\Sigma(x):=\sum_{g \in G_{\beta}} g(x) .
$$


It is easy to see that $\Sigma(x) \in \mathfrak{g}^{\bar{G}}$ and

$$
[H, \Sigma(x)]=\sum_{g \in G_{\beta}} g(\beta)(H) g(x)=h(\beta)(H) \sum_{g \in G_{\beta}} g(x)=h(\beta)(H) \Sigma(x)
$$

for all $H \in \mathcal{H}^{\bar{G}}$, since $h(g(\beta))=h(\beta)$ for any $g \in G$. It follows that $\Sigma(x)$ lies in the weight space $\left(\mathfrak{g}^{\bar{G}}\right)_{h(\beta)}$. Note that each element in $\mathfrak{g}^{\bar{G}}$ can be written as a sum of some $\Sigma(x)$ with $x \in \mathfrak{g}_{\beta}, \beta \in \Delta_{\widehat{Q}}$, we obtain that $\mathfrak{g}^{\bar{G}}$ is $\mathfrak{h}(\Gamma)$-diagonalisable.

Secondly, it is easy to see that the non-zero weights of $\mathfrak{g}^{\bar{G}}$ must be roots of $\Gamma$ since $h\left(\Delta_{\widehat{Q}}\right)=\Delta_{\Gamma}$. On the other hand, every root of $\Gamma$ is also a weight of $\mathfrak{g}^{\bar{G}}$ under the adjoint action by the monomorphism $\mathfrak{g}(\Gamma) \rightarrow \mathfrak{g}^{\bar{G}}$.

Finally, for any $\beta \in \Delta_{\Gamma}$, the set $\left\{\beta+k \bar{\varepsilon}_{i} \mid k \in \mathbb{Z}\right\} \cap \Delta_{\Gamma}$ is finite. Thus the action of $\bar{E}_{i}$ and $\bar{F}_{i}$ are local nilpotent on $\mathfrak{g}^{\bar{G}}$. The proof is completed.

Following from the proof of Proposition $4.6,\left(\mathfrak{g}^{\bar{G}}\right)_{\alpha}$ is spanned by the elements $\Sigma(x)=\sum_{g \in G_{\beta}} g(x)$, where $x \in \mathfrak{g}_{\beta}$ satisfies $g(x)=x$ for any $g \in H_{\beta}$, and $\beta \in \Delta_{\widehat{Q}}$ satisfies $h(\beta)=\alpha$. Thus, by the action of $G$ on $\left\{E_{i \rho}\right\}$ and $\left\{F_{i \rho}\right\}$, the action of $H_{\beta}$ on $\mathfrak{g}_{\beta}$ is identity and so that

$$
\operatorname{dim}_{\mathbb{k}}\left(\mathfrak{g}^{\bar{G}}\right)_{h(\beta)}=1
$$

for any simple $\operatorname{root} \beta$. That is, $\operatorname{dim}_{\mathbb{k}}\left(\mathfrak{g}^{\bar{G}}\right)_{\alpha}=1$ for all simple root $\alpha \in \Delta_{\Gamma}$. Moreover, we have the following claim.

Claim 4.7. $\operatorname{dim}_{\mathbb{k}}\left(\mathfrak{g}^{\bar{G}}\right)_{\alpha}=1$ for any real root $\alpha \in \Delta_{\Gamma}$.

Proof. We consider the automorphism

$$
\bar{r}_{i \rho}:=\exp \left(\operatorname{ad} F_{i \rho}\right) \exp \left(-\operatorname{ad} E_{i \rho}\right) \exp \left(\operatorname{ad} F_{i \rho}\right)
$$

of $\mathfrak{g}$. Then $\bar{r}_{i \rho}\left(\mathfrak{g}_{\beta}\right)=\mathfrak{g}_{r_{i \rho}(\beta)}$ and $\bar{r}_{i \rho}(H)=H-\varepsilon_{i \rho}(H) H_{i \rho}$ for any $H \in \mathfrak{h}$ (see [13, Lemma 3.8]). Note that $\bar{r}_{i \rho}$ and $\bar{r}_{i \rho^{\prime}}$ commute for any $\rho, \rho^{\prime} \in \operatorname{irr} G_{i}$, we let

$$
\bar{S}_{i}:=\prod_{\rho \in \operatorname{irr} G_{i}} \bar{r}_{i \rho} .
$$

Then, for any $H \in \mathcal{H}^{\bar{G}}$, we have

$$
\bar{S}_{i}(H)=H-\sum_{\rho \in \operatorname{irr} G_{i}} \varepsilon_{i \rho}(H) H_{i \rho}=H-\epsilon_{i}(H) \sum_{\rho \in \operatorname{irr} G_{i}} H_{i \rho}=H-\epsilon_{i}(H) H_{i},
$$

and $\bar{S}_{i}(H) \in \mathcal{H}^{\bar{G}}$. Note that $\bar{S}_{i}$ and $G$ commute on $\mathfrak{g}^{\prime}$, it deduces that $\bar{S}_{i}$ can define an automorphism of $\mathfrak{g}^{\bar{G}}$ such that

$$
\bar{S}_{i}\left(\left(\mathfrak{g}^{\bar{G}}\right)_{\alpha}\right)=\left(\mathfrak{g}^{\bar{G}}\right)_{\widehat{S}_{i}(\alpha)} .
$$

Thus, $\bar{S}_{i}$ is an extension of the automorphism $\exp \left(\operatorname{ad} \bar{F}_{i}\right) \exp \left(-\operatorname{ad} \bar{E}_{i}\right) \exp \left(\operatorname{ad} \bar{F}_{i}\right)$ of $\mathfrak{g}(\Gamma)$.

Let $\alpha \in \Delta_{\Gamma}$ be a real root. By Lemma 3.11 and Proposition 3.12, there exist a real root $\beta \in \Delta_{\widehat{Q}}$ and $\omega \in C_{G}(\mathcal{W}(\widehat{Q}))$ such that $h(\beta)=\alpha, \omega(\beta)$ is a 
simple root and $H_{\omega(\beta)}=H_{\beta}$. Let $\omega=\widehat{S}_{i_{1}} \widehat{S}_{i_{2}} \cdots \widehat{S}_{i_{r}}$ and $\bar{\omega}=\bar{S}_{i_{1}} \bar{S}_{i_{2}} \cdots \bar{S}_{i_{r}}$, then $\bar{\omega}\left(\mathfrak{g}_{\beta}\right)=\mathfrak{g}_{\omega(\beta)}$ and hence $\mathfrak{g}_{\beta}$ is fixed by $H_{\beta}$. Finally, note that all these $\beta$ are in the same $G$-orbit, we have $\operatorname{dim}_{\mathbb{k}}\left(\mathfrak{g}^{\bar{G}}\right)_{\alpha}=1$.

In particular, if $Q$ is a finite union of Dynkin quivers, then $\mathfrak{g}$ is a direct sum of simple Lie algebras and all roots of $\Gamma$ are real. By the claim, we have:

Corollary 4.8. If $Q$ is a finite union of Dynkin quivers and $G \subseteq \operatorname{Aut}(\mathbb{k} Q)$ is finite abelian, then there is a Lie algebra isomorphism $\mathfrak{g}(\Gamma) \cong \mathfrak{g}^{\bar{G}}$.

\section{Examples}

In this section, we give two examples to elucidate our results.

Example 5.1. Let $Q=(I, E)$ be the quiver:

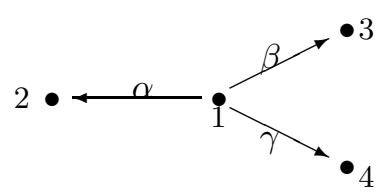

The action of $G=\langle g\rangle \cong \mathbb{Z} / 6 \mathbb{Z}$ on $\mathbb{k} Q$ given by

$$
\begin{array}{l|lllllll} 
& e_{1} & e_{2} & e_{3} & e_{4} & \alpha & \beta & \gamma \\
\hline g & e_{1} & e_{3} & e_{4} & e_{2} & -\beta & -\gamma & -\alpha
\end{array}
$$

where $e_{i}$ is the idempotent element of $\mathbb{k} Q$ corresponding to vertex $i, i \in$ $\{1,2,3,4\}$. Then the Cartan matrix of $Q$ is

$$
A=\left(a_{i j}\right)=\left(\begin{array}{cccc}
2 & -1 & -1 & -1 \\
-1 & 2 & 0 & 0 \\
-1 & 0 & 2 & 0 \\
-1 & 0 & 0 & 2
\end{array}\right) .
$$

Let $\varepsilon_{1}, \varepsilon_{2}, \varepsilon_{3}, \varepsilon_{4}$ be all the simple roots of the symmetric Kac-Moody algebra $\mathfrak{g}(Q)$. We endow the root lattice $\mathbb{Z} I$ with a symmetric bilinear form $(-,-)_{Q}$ via $\left(\varepsilon_{i}, \varepsilon_{j}\right)_{Q}=a_{i j}$ and define reflection $r_{i}: \alpha \mapsto \alpha-\left(\alpha, \varepsilon_{i}\right)_{Q} \varepsilon_{i}$ for each vertex $i \in I$. Then, it is well-known that Weyl group $\mathcal{W}(Q) \cong(\mathbb{Z} / 2 \mathbb{Z})^{3} \rtimes S_{4}$, and one can check that $\Delta_{Q}= \pm\left\{\varepsilon_{1}, \varepsilon_{2}, \varepsilon_{3}, \varepsilon_{4}, \varepsilon_{1}+\varepsilon_{2}, \varepsilon_{1}+\varepsilon_{3}, \varepsilon_{1}+\varepsilon_{4}, \varepsilon_{1}+\varepsilon_{2}+\varepsilon_{3}, \varepsilon_{1}+\right.$ $\left.\varepsilon_{2}+\varepsilon_{4}, \varepsilon_{1}+\varepsilon_{3}+\varepsilon_{4}, \varepsilon_{1}+\varepsilon_{2}+\varepsilon_{3}+\varepsilon_{4}, 2 \varepsilon_{1}+\varepsilon_{2}+\varepsilon_{3}+\varepsilon_{4}\right\}$ is the root system of $\mathfrak{g}(Q)$.

We get the generalized McKay quiver $\widehat{Q}=(\widehat{I}, \widehat{E})$ of $(Q, G)$ as follows:
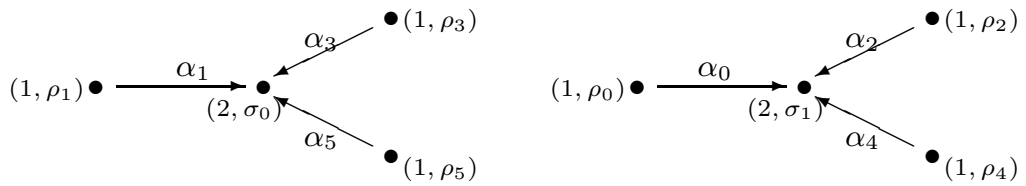

where $\rho_{i}$ is the irreducible representation of $G=\langle g\rangle \cong \mathbb{Z} / 6 \mathbb{Z}$ defined by

$$
a \cdot g=\xi^{i} a, \quad a \in \rho_{i},
$$


$\sigma_{j}$ is the irreducible representation of $\left\langle g^{3}\right\rangle \cong \mathbb{Z} / 2 \mathbb{Z}$ defined by

$$
b \cdot g^{3}=\xi^{3 j} b, \quad b \in \sigma_{j},
$$

and $\xi$ is a primitive 6 -th root of unity. As we have discussed in Section 3.2, by the group isomorphism

$$
\varphi: G \rightarrow \widetilde{G}, \quad \varphi\left(g^{i}\right)=\chi_{g^{i}}, \quad \chi_{g^{i}}\left(g^{j}\right)=\xi^{i j},
$$

we define the action of $G$ on $\mathbb{k} Q * G$ by setting

$$
g^{i}\left(\lambda g^{j}\right)=\xi^{i j} \lambda g^{j}
$$

for any $g^{i} \in G, \lambda g^{j} \in \mathbb{k} Q * G$. This induces an action of $G=\langle g\rangle \cong \mathbb{Z} / 6 \mathbb{Z}$ on $\mathbb{k} \widehat{Q}$ given by

\begin{tabular}{l|llllllllllllll} 
& $e_{0}$ & $e_{1}$ & $e_{2}$ & $e_{3}$ & $e_{4}$ & $e_{5}$ & $e_{0}^{\prime}$ & $e_{1}^{\prime}$ & $\alpha_{0}$ & $\alpha_{1}$ & $\alpha_{2}$ & $\alpha_{3}$ & $\alpha_{4}$ & $\alpha_{5}$ \\
\hline$g$ & $e_{1}$ & $e_{2}$ & $e_{3}$ & $e_{4}$ & $e_{5}$ & $e_{0}$ & $e_{1}^{\prime}$ & $e_{0}^{\prime}$ & $\xi_{0} \alpha_{1}$ & $\xi_{1} \alpha_{2}$ & $\xi_{2} \alpha_{3}$ & $\xi_{3} \alpha_{4}$ & $\xi_{4} \alpha_{5}$ & $\xi_{5} \alpha_{0}$
\end{tabular} where idempotent elements $e_{i}, e_{i}^{\prime}$ are corresponding to the vertices $\left(1, \rho_{i}\right),\left(2, \sigma_{i}\right)$ respectively, and $\xi_{i} \in \mathbb{k}$ satisfying $\xi_{0} \xi_{1} \cdots \xi_{5}=1$. One can check that the generalized McKay quiver of $(\widehat{Q}, G)$ is just the quiver $Q$.

By the definition given in Section 2.3, we obtain the symmetrisable generalized Cartan matrix $C$ corresponding to $(Q, G)$, i.e.,

$$
C=\left(\begin{array}{cc}
2 & -1 \\
-3 & 2
\end{array}\right)
$$

Then the valued graph $\Gamma$ corresponding to $C$ is

$$
\Gamma: \quad 1 \bullet \frac{(3,1)}{\bullet} \bullet 2
$$

Let $\bar{\varepsilon}_{1}, \bar{\varepsilon}_{2}$ be all the simple roots of $\Gamma$. Then the Weyl group

$$
\mathcal{W}(\Gamma) \cong D_{6}=\left\langle a, b \mid a^{2}=1, b^{3}=1, a b=b^{-1} a\right\rangle
$$

and root system $\Delta_{\Gamma}=\left\{\bar{\varepsilon}_{1}, \bar{\varepsilon}_{2}, \bar{\varepsilon}_{1}+\bar{\varepsilon}_{2}, 2 \bar{\varepsilon}_{1}+\bar{\varepsilon}_{2}, 3 \bar{\varepsilon}_{1}+\bar{\varepsilon}_{2}, 3 \bar{\varepsilon}_{1}+2 \bar{\varepsilon}_{2}\right\}$. See Section 2.3 for detail.

We consider the map

$$
h: \quad \mathbb{Z} \widehat{I} \longrightarrow \mathbb{Z I}, \quad h(\alpha)_{i}=\sum_{\rho \in \operatorname{irr} G_{i}} \alpha_{i \rho}
$$

for any $\alpha=\sum_{(i, \rho) \in \widehat{I}} \alpha_{i \rho} \varepsilon_{(i \rho) \in \widehat{I}} \in \mathbb{Z} \widehat{I}$. The restriction of $h: \Delta_{\widehat{Q}} \rightarrow \Delta_{\Gamma}$ is surjective, this means that for any positive root $\beta$ of $\Gamma$, there exists an indecomposable $\widehat{Q}$-representation $X$ such that $h(\operatorname{dim} X)=\beta$. For example, we consider the positive root $\bar{\varepsilon}_{1}+\bar{\varepsilon}_{2} \in \Delta_{\Gamma}$. Then, we have the following indecomposable $\widehat{Q}$-representation $X_{\left(\rho_{3} \sigma_{0}\right)}$ :
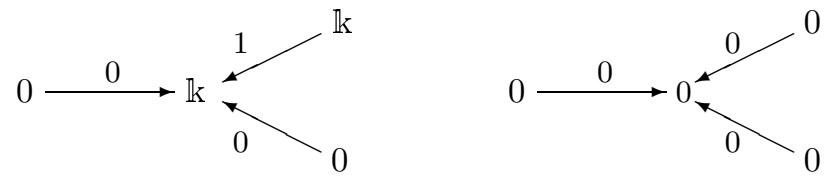
and obviously, $h\left(\operatorname{dim} X_{\left(\rho_{3} \sigma_{0}\right)}\right)=\bar{\varepsilon}_{1}+\bar{\varepsilon}_{2}$. Furthermore, for any $0 \leq l \leq 5$, $0 \leq j \leq 1$ and $l \not \equiv j \bmod 2$, we define the $\widehat{Q}$-representation $X_{\left(\rho_{l} \sigma_{j}\right)}=\left(X_{i \rho}, X_{\alpha}\right)$ by

$$
X_{i \rho}= \begin{cases}\mathbb{k}, & \text { if }(i, \rho)=\left(1, \rho_{l}\right) \text { or }\left(2, \sigma_{j}\right) ; \\ 0, & \text { otherwise. }\end{cases}
$$

Then, it is easy to see that the set of all indecomposable $\widehat{Q}$-representations with $h(\operatorname{dim} X)=\bar{\varepsilon}_{1}+\bar{\varepsilon}_{2}$ is the set

$$
\left\{X_{\left(\rho_{l} \sigma_{j}\right)} \mid 0 \leq l \leq 5,0 \leq j \leq 1 \text { and } l \not \equiv j \bmod 2\right\},
$$

and which is just the orbit of $X_{\left(\rho_{1} \sigma_{0}\right)}$ under that action of $G$. Similarly, for any positive real root $\beta=h(\alpha) \in \Delta_{\Gamma}$, there are $\left|H_{\alpha}\right|$ (up to isomorphism) indecomposable $\widehat{Q}$-representations $X$ such that $h(\operatorname{dim} X)=\beta$.

Example 5.2. Let $Q=(I, E)$ be the quiver
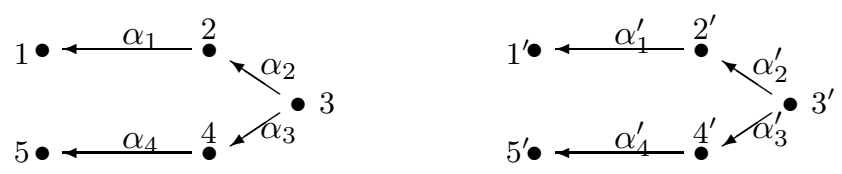

and $G=\langle a\rangle \times\langle b\rangle \cong \mathbb{Z} / 2 \mathbb{Z} \times \mathbb{Z} / 2 \mathbb{Z}$. The action of $G$ on $\mathbb{k} Q$ is given as follows

\begin{tabular}{c|cccccccccc} 
& $e_{1}$ & $e_{2}$ & $e_{3}$ & $e_{4}$ & $e_{5}$ & $e_{1^{\prime}}$ & $e_{2^{\prime}}$ & $e_{3^{\prime}}$ & $e_{4^{\prime}}$ & $e_{5^{\prime}}$ \\
\hline$a$ & $e_{5}$ & $e_{4}$ & $e_{3}$ & $e_{2}$ & $e_{1}$ & $e_{5^{\prime}}$ & $e_{4^{\prime}}$ & $e_{3^{\prime}}$ & $e_{2^{\prime}}$ & $e_{1^{\prime}}$ \\
$b$ & $e_{1^{\prime}}$ & $e_{2^{\prime}}$ & $e_{3^{\prime}}$ & $e_{4^{\prime}}$ & $e_{5^{\prime}}$ & $e_{1}$ & $e_{2}$ & $e_{3}$ & $e_{4}$ & $e_{5}$ \\
& & $\alpha_{1}$ & $\alpha_{2}$ & $\alpha_{3}$ & $\alpha_{4}$ & $\alpha_{1}^{\prime}$ & $\alpha_{2}^{\prime}$ & $\alpha_{3}^{\prime}$ & $\alpha_{4}^{\prime}$ \\
\hline & $a$ & $\alpha_{4}$ & $\alpha_{3}$ & $\alpha_{2}$ & $\alpha_{1}$ & $\alpha_{4}^{\prime}$ & $\alpha_{3}^{\prime}$ & $\alpha_{2}^{\prime}$ & $\alpha_{1}^{\prime}$ \\
& $b$ & $\alpha_{1}^{\prime}$ & $\alpha_{2}^{\prime}$ & $\alpha_{3}^{\prime}$ & $\alpha_{4}^{\prime}$ & $\alpha_{1}$ & $\alpha_{2}$ & $\alpha_{3}$ & $\alpha_{4}$
\end{tabular}

where $e_{i}$ is the idempotent element of $\mathbb{k} Q$ corresponding to the vertex $i$. Take $\mathcal{I}=\{1,2,3\}$. Then the generalized McKay quiver of $(Q, G)$ is

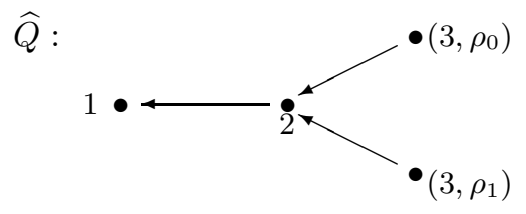

where $\rho_{0}, \rho_{1}$ are the non-isomorphism irreducible representations of $G_{3}=\langle a\rangle \cong$ $\mathbb{Z} / 2 \mathbb{Z}$. Reindexing the vertex set $\widehat{I}=\left\{1,2,\left(3, \rho_{0}\right),\left(3, \rho_{1}\right)\right\}$ by $\{1,2,3,4\}$, the Cartan matrix of $\widehat{Q}$ is

$$
A=\left(a_{i j}\right)=\left(\begin{array}{cccc}
2 & -1 & 0 & 0 \\
-1 & 2 & -1 & -1 \\
0 & -1 & 2 & 0 \\
0 & -1 & 0 & 2
\end{array}\right) .
$$


The Lie algebra $\mathfrak{g}:=\mathfrak{g}(\widehat{Q})$ is generated by $\left\{x_{i}, y_{i}, h_{i} \mid 1 \leq i \leq 4\right\}$ satisfying the relations

$$
\begin{array}{ll}
{\left[h_{i}, h_{j}\right]=0,} & {\left[x_{i}, y_{j}\right]=\delta_{i j} h_{i} ;} \\
{\left[h_{i}, x_{j}\right]=a_{i j} x_{j},} & {\left[h_{i}, y_{j}\right]=-a_{i j} y_{j} ;} \\
\left(\operatorname{ad} x_{i}\right)^{1-a_{i j}}\left(x_{j}\right)=0, & \left(\operatorname{ad} y_{i}\right)^{1-a_{i j}}\left(y_{j}\right)=0, \quad i \neq j .
\end{array}
$$

In this case, the valued graph $\Gamma$ of $(Q, G)$ is

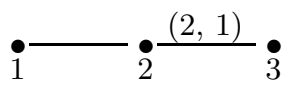

with the Cartan Matrix

$$
C=\left(\begin{array}{ccc}
2 & -1 & 0 \\
-1 & 2 & -1 \\
0 & -2 & 2
\end{array}\right)
$$

The Lie algebra $\mathfrak{g}(\Gamma)$ is generated by $\left\{X_{i}, Y_{i}, H_{i} \mid 1 \leq i \leq 3\right\}$ satisfying the relations

$$
\begin{array}{lll}
{\left[H_{i}, H_{j}\right]=0,} & {\left[X_{i}, Y_{j}\right]=\delta_{i j} H_{i} ;} \\
{\left[H_{i}, X_{j}\right]=c_{i j} X_{j},} & {\left[H_{i}, Y_{j}\right]=-c_{i j} Y_{j} ;} \\
\left(\operatorname{ad} X_{i}\right)^{1-c_{i j}}\left(X_{j}\right)=0, & \left(\operatorname{ad} Y_{i}\right)^{1-c_{i j}}\left(Y_{j}\right)=0, \quad i \neq j .
\end{array}
$$

As the discussion in Section 3.2, we see that the vertices $\left(3, \rho_{0}\right)$ and $\left(3, \rho_{1}\right)$ of $\widehat{Q}$ are in the same $G$-orbit. Therefore, the Lie algebra $\mathfrak{g}^{\bar{G}}$ is generated by

$$
\left\{\bar{x}_{i}, \bar{y}_{i}, \bar{h}_{i} \mid 1 \leq i \leq 3\right\}
$$

where $\bar{x}_{i}=x_{i}, \bar{y}_{i}=y_{i}, \bar{h}_{i}=h_{i}$ for $i=1,2$, and $\bar{x}_{3}=x_{3}+x_{4}, \bar{y}_{3}=y_{3}+y_{4}$, $\bar{h}_{3}=h_{3}+h_{4}$, satisfying the relations (1). Then, it is easy to see that the map

$$
\Phi: \quad \mathfrak{g}(\Gamma) \longrightarrow \mathfrak{g}^{\bar{G}}
$$

given by

$$
\Phi\left(X_{i}\right)=\bar{x}_{i}, \quad \Phi\left(Y_{i}\right)=\bar{y}_{i}, \quad \Phi\left(H_{i}\right)=\bar{h}_{i}
$$

is a Lie algebra isomorphism.

At last, we consider the following Dynkin quivers:

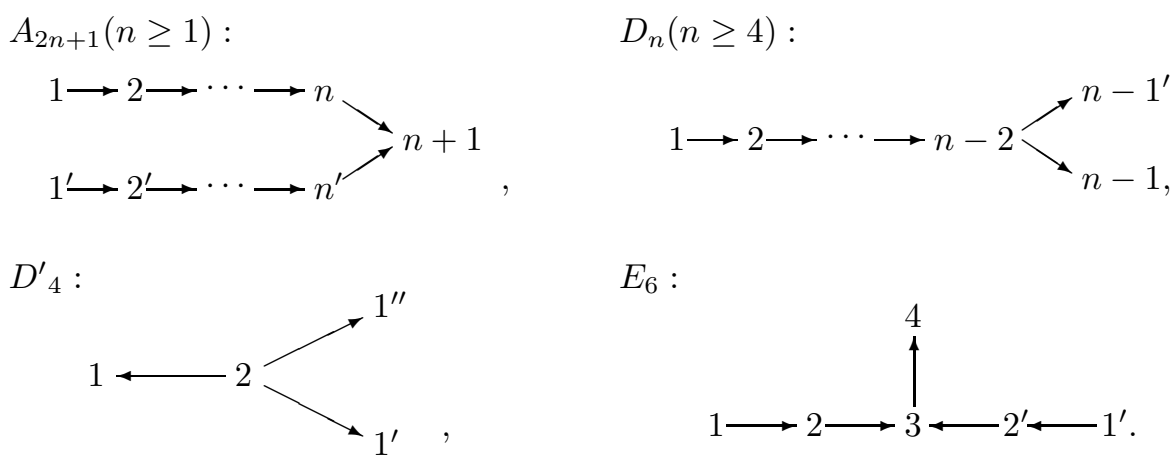


We equip the quivers $A_{2 n+1}, D_{n}$ and $E_{6}$ with the quiver automorphism group $G=\mathbb{Z} / 2 \mathbb{Z}$. For the quiver $D^{\prime}{ }_{4}$, we consider its quiver automorphism group $G=\mathbb{Z} / 3 \mathbb{Z}$. Then, we have

\begin{tabular}{|c|c|c|c|c|c|}
\hline$Q$ & $G$ & $\Gamma$ & $\widehat{Q}$ & $\widehat{\Gamma}$ & Conclusion \\
\hline$A_{2 n+1}$ & $\mathbb{Z} / 2 \mathbb{Z}$ & $B_{n+1}$ & $D_{n+2}$ & $C_{n+1}$ & $\mathfrak{g}\left(B_{n+1}\right) \cong \mathfrak{g}\left(D_{n+2}\right)^{\mathbb{Z} / 2 \mathbb{Z}}$ \\
\hline$D_{n}$ & $\mathbb{Z} / 2 \mathbb{Z}$ & $C_{n-1}$ & $A_{2 n-1}$ & $B_{n-1}$ & $\mathfrak{g}\left(C_{n-1}\right) \cong \mathfrak{g}\left(A_{2 n-1}\right)^{\mathbb{Z} / 2 \mathbb{Z}}$ \\
\hline$D^{\prime}{ }_{4}$ & $\mathbb{Z} / 3 \mathbb{Z}$ & $G_{2}$ & $D^{\prime \text { op }}$ & $G_{2}$ & $\mathfrak{g}\left(G_{2}\right) \cong \mathfrak{g}\left(D^{\prime}{ }_{4}\right)^{\mathbb{Z} / 3 \mathbb{Z}}$ \\
\hline$E_{6}$ & $\mathbb{Z} / 2 \mathbb{Z}$ & $F_{4}$ & $E_{6}^{\text {op }}$ & $F_{4}$ & $\mathfrak{g}\left(F_{4}\right) \cong \mathfrak{g}\left(E_{6}\right)^{\mathbb{Z} / 2 \mathbb{Z}}$ \\
\hline
\end{tabular}

where $D_{4}^{\prime \text { op }}$ and $E_{6}^{\text {op }}$ are the opposite quiver of $D^{\prime}{ }_{4}$ and $E_{6}$, respectively. $B_{n}$, $C_{n}, F_{4}$ and $G_{2}$ mean the $B$-type, $C$-type, $F$-type and $G$-type Dynkin graph respectively (see [7]).

We conclude that all the finite-dimensional simple Lie algebras corresponding to the Dynkin graphs with multiple edges can be realized by the fix point algebras of the simple Lie algebras corresponding to the Dynkin graphs without multiple edges.

\section{References}

[1] I. Assem, D. Simson, and A. Skowroński, Elements of Representation Theory of Associative Algebras. Vol. 1, Cambridge University Press, 2006.

[2] M. Auslander, Rational singularities and almost split sequences, Trans. Amer. Math. Soc. 293 (1986), no. 2, 511-531.

[3] M. Auslander, I. Reiten, and S. O. Smalo, Representation Theory of Artin Algebras, Cambridge Stud. Adv. Math., Vol. 36, Cambridge University Press, 1995.

[4] W. Crawley-Boevey and M. P. Holland, Noncommutative deformations of Kleinian singularities, Duke Math. J. 92 (1998), no. 3, 605-635.

[5] L. Demonet, Skew group algebras of path algebras and preprojective algebras, J. Algebra 323 (2010), no. 4, 1052-1059.

[6] B. Deng, J. Du, B. Parshall, and J. Wang, Finite Dimensional Algebras and Puantum Groups, Math. Surveys and Monographs 150, American Mathematical Society, Providence, 2008.

[7] V. Dlab and C. M. Ringel, Indecomposable representations of graphs and algebras, Mem. Amer. Math. Soc. 6 (1976), no. 173, 57 pp.

[8] J. Guo, On the McKay quivers and m-Cartan Matrices, Sci. China Ser. A 52 (2009), no. $3,511-516$.

[9] J. Guo and Martiínez-Villa, Algebra pairs associated to McKay quivers, Comm. Algebra 30 (2002), no. 2, 1017-1032.

[10] B. Hou and S. Yang, Skew group algebras of deformed preprojective algebras, J. Algebra 332 (2011), 209-228.

[11] A. Hubery, Representations of quiver respecting a quiver automorphism and a of Kac, $\mathrm{Ph}$. D. thesis, Leeds Univeraity, 2002.

[12] — Quiver representations respecting a quiver automorphism: a generalisation of a theorem of Kac, J. London Math. Soc. 69 (2004), no. 1, 79-96.

[13] V. G. Kac, Infinite Dimensional Lie Algebras, 3rd edn, Cambridge University Press, Cambridge, 1990. 
[14] V. G. Kac and S. P. Wang, On automorphisms of Kac-Moody algebras and groups, Adv. Math. 92 (1992), no. 2, 129-195

[15] G. Lusztig, Affine quivers and canonical bases, Inst. Hautes Études Sci. Publ. Math. 76 (1992), 111-163.

[16] J. McKay, Graphs, singularities and finite groups, Proc. Sympos. Pure Math., vol. 37, pp. 183-186, Amer. Math. Soc., Providence, RI, 1980.

[17] M. Reid, McKay correspondance, arXiv:math.AG/9702016.

[18] I. Reiten and C. Riedtmann, Skew group algebras in the representation theory of Artin algebras, J. Algebra 92 (1985), no. 1, 224-282.

Bo Hou

School of Mathematics and Statistics

HENAN University

KAIFENG 475001, P. R. ChINA

E-mail address: bohou1981@163.com

Shilin Yang

College of Applied Science

Beijing University of Technology

Beijing 100124, P. R. China

E-mail address: slyang@bjut.edu.cn 